\title{
Renormalization group evolution of the Standard Model dimension six operators II: Yukawa dependence
}

\author{
Elizabeth E. Jenkins, ${ }^{a}$ Aneesh V. Manohar ${ }^{a}$ and Michael Trott ${ }^{b, 1}$ \\ ${ }^{a}$ Department of Physics, University of California at San Diego, \\ 9500 Gilman Drive, La Jolla, CA 92093-0319, U.S.A. \\ ${ }^{b}$ Theory Division, Physics Department, CERN, \\ CH-1211 Geneva 23, Switzerland \\ E-mail: ejenkins@ucsd.edu, amanohar@ucsd.edu, michael.trott@cern.ch
}

\begin{abstract}
We calculate the complete order $y^{2}$ and $y^{4}$ terms of the $59 \times 59$ one-loop anomalous dimension matrix for the dimension-six operators of the Standard Model effective field theory, where $y$ is a generic Yukawa coupling. These terms, together with the terms of order $\lambda, \lambda^{2}$ and $\lambda y^{2}$ depending on the Standard Model Higgs self-coupling $\lambda$ which were calculated in a previous work, yield the complete one-loop anomalous dimension matrix in the limit of vanishing gauge couplings. The Yukawa contributions result in non-trivial flavor mixing in the various operator sectors of the Standard Model effective theory.
\end{abstract}

Keywords: Renormalization Group, Higgs Physics, Beyond Standard Model, Standard Model

ARXIV EPRINT: 1310.4838

\footnotetext{
${ }^{1}$ Corresponding author.
} 


\section{Contents}

1 Introduction 1

2 Formalism 2

3 Discussion 5

4 Conclusion $\quad 7$

$\begin{array}{lll}\text { A Results } & 7\end{array}$

A.1 $X^{3} \quad 8$

A.2 $H^{6}$

A.3 $H^{4} D^{2}$

$\begin{array}{lll}\text { A.4 } X^{2} H^{2} & 9\end{array}$

A.5 $\psi^{2} H^{3}$

A.6 $\psi^{2} X H \quad 10$

A.7 $\psi^{2} H^{2} D=10$

$\begin{array}{lll}\text { A.8 } & \psi^{4} & 11\end{array}$

A.8.1 $(\bar{L} L)(\bar{L} L) \quad 11$

$\begin{array}{lll}\text { A.8.2 } & (\bar{R} R)(\bar{R} R) & 12\end{array}$

A.8.3 $(\bar{L} L)(\bar{R} R) \quad 13$

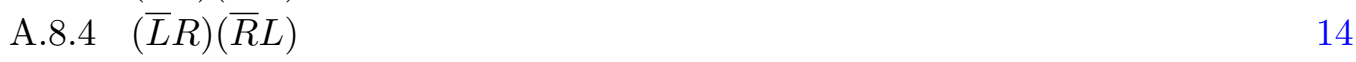

A.8.5 $(\bar{L} R)(\bar{L} R) \quad 14$

\section{Introduction}

The LHC has discovered a Higgs-like boson with properties consistent with Standard Model (SM) predictions. In addition, the SM provides a successful description of experimental data up to energies $v=246 \mathrm{GeV}$, the scale of electroweak symmetry breaking, and there is no evidence thus far for any additional particles beyond the SM. It is important to study the properties of the Higgs boson to high precision, and to increase the energy at the LHC to search for additional particles or phenomena at higher energy. A widely used approach to studying new physics in light of the current experimental situation is to generalize the SM to an effective field theory (EFT) by adding higher dimensional (non-renormalizable) operators constructed out of SM fields to the SM Lagrangian. This approach implicitly assumes that $\mathrm{SU}(2)_{L} \times \mathrm{U}(1)_{Y}$ is a linearly realized symmetry in the scalar sector, which is an assumption we adopt in this work. The non-renormalizable operators are suppressed by an energy scale $\Lambda>v$, and they parametrize the low-energy effects of new physics at energies above $\Lambda$. In the effective field theory approach, higher dimensional operators yield 
effects which are ordered in a power series expansion in $E / \Lambda$. Operators of mass dimension $d$ in the SM EFT yield effects which are order $(E / \Lambda)^{d-4}$. Thus, the largest effects for $E<\Lambda$ arise from the non-renormalizable operators of smallest mass dimension.

The leading operators which preserve lepton number first arise at dimension six, and have been classified in refs. $[1,2]$. There are 59 independent dimension-six operators which preserve baryon number after redundant operators have been eliminated by field redefinitions, or equivalently, by using the equations of motion (EOM). These 59 operators divide into eight operator classes, labelled by their field content and number of covariant derivatives. Denoting gauge field strengths by $X=G_{\mu \nu}, W_{\mu \nu}, B_{\mu \nu}$, the Higgs doublet scalar field by $H$, fermion fields by $\psi=q, u, d, l$, e, and covariant derivatives by $D$, the eight operator classes are $1: X^{3}, 2: H^{6}, 3: H^{4} D^{2}, 4: X^{2} H^{2}, 5: \psi^{2} H^{3}, 6: \psi^{2} X H, 7: \psi^{2} H^{2} D$ and $8: \psi^{4}$. Since we make extensive use of these operators, we list them again in table 1 .

The anomalous dimensions of the dimension-six operators enter into Higgs phenomenology. In ref. [3], we computed the anomalous dimension matrix of the eight $X^{2} H^{2}$ operators which contribute to $h \rightarrow \gamma \gamma, h \rightarrow \gamma Z$ and $g g \rightarrow h$, which are crucial processes for precision Higgs experiments, and in [4] an exactly solvable model was constructed that generates precisely these operators. In ref. [5], we embarked on the calculation of the complete one-loop anomalous dimension matrix for the 59 independent dimension-six operators. In addition, we calculated all contributions to the running of the $d \leq 4$ SM parameters from the 59 independent dimension-six operators. The contributions to the running of SM parameters from dimension-six operators is order $v^{2} / \Lambda^{2}$, which is as important as the tree-level contribution of dimension-six operators.

The present paper continues our computation of the one-loop anomalous dimension matrix of the dimension-six operators. This matrix, with $59 \times 59=3481$ entries, can be broken into block submatrices $\gamma_{i j}$ where $i, j=1, \cdots, 8$ label the eight operator classes. In this paper, we present the Yukawa terms, leaving the gauge terms for a subsequent publication. The Yukawa terms contribute to flavor-changing processes. The anomalous dimensions we compute can give flavor-changing Higgs couplings to fermions, and they can lead to rare decays such as $\mu \rightarrow e \gamma$. The anomalous dimension calculation involves a large number $(\sim 100)$ one-loop diagrams, a selection of which are shown in figure 1. Each graph is simple to compute, but computing the full flavor dependence is tedious. For example, a single graph in figure 1 for the $\psi^{4}-\psi^{4}$ mixing of four-fermion operators into themselves leads to a set of anomalous dimensions that occupy seven pages of the appendix.

Some aspects of operator mixing of dimension-six operators due to Yukawa couplings has been previously calculated in refs. [6-11]. In particular, ref. [11] recently studied operator mixing from a different viewpoint, and calculated, for a single flavor, some entries in rows 3,5 and 6 of the anomalous dimension matrix, including the gauge dependence.

\section{Formalism}

The Lagrangian of the SM EFT is given by

$$
\mathcal{L}=\mathcal{L}_{S M}+\sum_{d>4} \mathcal{L}^{(d)}
$$




\begin{tabular}{|c|c|}
\hline \multicolumn{2}{|c|}{$1: X^{3}$} \\
\hline$Q_{G}$ & $f^{A B C} G_{\mu}^{A \nu} G_{\nu}^{B \rho} G_{\rho}^{C \mu}$ \\
$Q_{\widetilde{G}}$ & $f^{A B C} \widetilde{G}_{\mu}^{A \nu} G_{\nu}^{B \rho} G_{\rho}^{C \mu}$ \\
$Q_{W}$ & $\epsilon^{I J K} W_{\mu}^{I \nu} W_{\nu}^{J \rho} W_{\rho}^{K \mu}$ \\
$Q_{\widetilde{W}}$ & $\epsilon^{I J K} \widetilde{W}_{\mu}^{I \nu} W_{\nu}^{J \rho} W_{\rho}^{K \mu}$ \\
\hline
\end{tabular}

\begin{tabular}{|c|c|}
\hline \multicolumn{2}{|c|}{$4: X^{2} H^{2}$} \\
\hline$Q_{H G}$ & $H^{\dagger} H G_{\mu \nu}^{A} G^{A \mu \nu}$ \\
$Q_{H \widetilde{G}}$ & $H^{\dagger} H \widetilde{G}_{\mu \nu}^{A} G^{A \mu \nu}$ \\
$Q_{H W}$ & $H^{\dagger} H W_{\mu \nu}^{I} W^{I \mu \nu}$ \\
$Q_{H \widetilde{W}}$ & $H^{\dagger} H \widetilde{W}_{\mu \nu}^{I} W^{I \mu \nu}$ \\
$Q_{H B}$ & $H^{\dagger} H B_{\mu \nu} B^{\mu \nu}$ \\
$Q_{H \widetilde{B}}$ & $H^{\dagger} H \widetilde{B}_{\mu \nu} B^{\mu \nu}$ \\
$Q_{H W B}$ & $H^{\dagger} \tau^{I} H W_{\mu \nu}^{I} B^{\mu \nu}$ \\
$Q_{H \widetilde{W} B}$ & $H^{\dagger} \tau^{I} H \widetilde{W}_{\mu \nu}^{I} B^{\mu \nu}$ \\
\hline
\end{tabular}

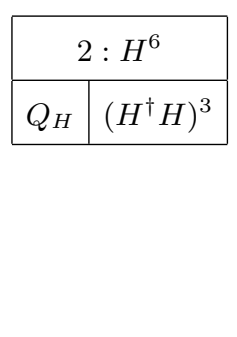

\begin{tabular}{|c|c|}
\hline \multicolumn{2}{|c|}{$5: \psi^{2} H^{3}+$ h.c. } \\
\hline$Q_{e H}$ & $\left(H^{\dagger} H\right)\left(\bar{l}_{p} e_{r} H\right)$ \\
$Q_{u H}$ & $\left(H^{\dagger} H\right)\left(\bar{q}_{p} u_{r} \widetilde{H}\right)$ \\
$Q_{d H}$ & $\left(H^{\dagger} H\right)\left(\bar{q}_{p} d_{r} H\right)$ \\
\hline
\end{tabular}

\begin{tabular}{|c|c|}
\hline \multicolumn{2}{|c|}{$6: \psi^{2} X H+$ h.c. } \\
\hline$Q_{e W}$ & $\left(\bar{l}_{p} \sigma^{\mu \nu} e_{r}\right) \tau^{I} H W_{\mu \nu}^{I}$ \\
$Q_{e B}$ & $\left(\bar{l}_{p} \sigma^{\mu \nu} e_{r}\right) H B_{\mu \nu}$ \\
$Q_{u G}$ & $\left(\bar{q}_{p} \sigma^{\mu \nu} T^{A} u_{r}\right) \tilde{H} G_{\mu \nu}^{A}$ \\
$Q_{u W}$ & $\left(\bar{q}_{p} \sigma^{\mu \nu} u_{r}\right) \tau^{I} \widetilde{H} W_{\mu \nu}^{I}$ \\
$Q_{u B}$ & $\left(\bar{q}_{p} \sigma^{\mu \nu} u_{r}\right) \tilde{H} B_{\mu \nu}$ \\
$Q_{d G}$ & $\left(\bar{q}_{p} \sigma^{\mu \nu} T^{A} d_{r}\right) H G_{\mu \nu}^{A}$ \\
$Q_{d W}$ & $\left(\bar{q}_{p} \sigma^{\mu \nu} d_{r}\right) \tau^{I} H W_{\mu \nu}^{I}$ \\
$Q_{d B}$ & $\left(\bar{q}_{p} \sigma^{\mu \nu} d_{r}\right) H B_{\mu \nu}$ \\
\hline
\end{tabular}

\begin{tabular}{|c|c|}
\hline \multicolumn{2}{|c|}{$7: \psi^{2} H^{2} D$} \\
\hline$Q_{H l}^{(1)}$ & $\left(H^{\dagger} i \overleftrightarrow{D_{\mu}} H\right)\left(\bar{l}_{p} \gamma^{\mu} l_{r}\right)$ \\
$Q_{H l}^{(3)}$ & $\left(H^{\dagger} i \overleftrightarrow{D}_{\mu}^{I} H\right)\left(\bar{l}_{p} \tau^{I} \gamma^{\mu} l_{r}\right)$ \\
$Q_{H e}$ & $\left(H^{\dagger} i \overleftrightarrow{D}_{\mu} H\right)\left(\bar{e}_{p} \gamma^{\mu} e_{r}\right)$ \\
$Q_{H q}^{(1)}$ & $\left(H^{\dagger} i \overleftrightarrow{D}_{\mu} H\right)\left(\bar{q}_{p} \gamma^{\mu} q_{r}\right)$ \\
$Q_{H q}^{(3)}$ & $\left(H^{\dagger} i \overleftrightarrow{D}_{\mu}^{I} H\right)\left(\bar{q}_{p} \tau^{I} \gamma^{\mu} q_{r}\right)$ \\
$Q_{H u}$ & $\left(H^{\dagger} i \overleftrightarrow{D}_{\mu} H\right)\left(\bar{u}_{p} \gamma^{\mu} u_{r}\right)$ \\
$Q_{H d}$ & $\left(H^{\dagger} i \overleftrightarrow{D}_{\mu} H\right)\left(\bar{d}_{p} \gamma^{\mu} d_{r}\right)$ \\
$Q_{H u d}+$ h.c. & $i\left(\widetilde{H}^{\dagger} D_{\mu} H\right)\left(\bar{u}_{p} \gamma^{\mu} d_{r}\right)$ \\
\hline
\end{tabular}

\begin{tabular}{|c|c|}
\hline \multicolumn{2}{|c|}{$8:(\bar{L} L)(\bar{L} L)$} \\
\hline$Q_{l l}$ & $\left(\bar{l}_{p} \gamma_{\mu} l_{r}\right)\left(\bar{l}_{s} \gamma^{\mu} l_{t}\right)$ \\
$Q_{q q}^{(1)}$ & $\left(\bar{q}_{p} \gamma_{\mu} q_{r}\right)\left(\bar{q}_{s} \gamma^{\mu} q_{t}\right)$ \\
$Q_{q q}^{(3)}$ & $\left(\bar{q}_{p} \gamma_{\mu} \tau^{I} q_{r}\right)\left(\bar{q}_{s} \gamma^{\mu} \tau^{I} q_{t}\right)$ \\
$Q_{l q}^{(1)}$ & $\left(\bar{l}_{p} \gamma_{\mu} l_{r}\right)\left(\bar{q}_{s} \gamma^{\mu} q_{t}\right)$ \\
$Q_{l q}^{(3)}$ & $\left(\bar{l}_{p} \gamma_{\mu} \tau^{I} l_{r}\right)\left(\bar{q}_{s} \gamma^{\mu} \tau^{I} q_{t}\right)$ \\
\hline
\end{tabular}

\begin{tabular}{|c|c|}
\hline \multicolumn{2}{|c|}{$8:(\bar{R} R)(\bar{R} R)$} \\
\hline$Q_{e e}$ & $\left(\bar{e}_{p} \gamma_{\mu} e_{r}\right)\left(\bar{e}_{s} \gamma^{\mu} e_{t}\right)$ \\
$Q_{u u}$ & $\left(\bar{u}_{p} \gamma_{\mu} u_{r}\right)\left(\bar{u}_{s} \gamma^{\mu} u_{t}\right)$ \\
$Q_{d d}$ & $\left(\bar{d}_{p} \gamma_{\mu} d_{r}\right)\left(\bar{d}_{s} \gamma^{\mu} d_{t}\right)$ \\
$Q_{e u}$ & $\left(\bar{e}_{p} \gamma_{\mu} e_{r}\right)\left(\bar{u}_{s} \gamma^{\mu} u_{t}\right)$ \\
$Q_{e d}$ & $\left(\bar{e}_{p} \gamma_{\mu} e_{r}\right)\left(\bar{d}_{s} \gamma^{\mu} d_{t}\right)$ \\
$Q_{u d}^{(1)}$ & $\left(\bar{u}_{p} \gamma_{\mu} u_{r}\right)\left(\bar{d}_{s} \gamma^{\mu} d_{t}\right)$ \\
$Q_{u d}^{(8)}$ & $\left(\bar{u}_{p} \gamma_{\mu} T^{A} u_{r}\right)\left(\bar{d}_{s} \gamma^{\mu} T^{A} d_{t}\right)$ \\
\hline
\end{tabular}

\begin{tabular}{|c|c|}
\hline \multicolumn{2}{|c|}{$8:(\bar{L} L)(\bar{R} R)$} \\
\hline$Q_{l e}$ & $\left(\bar{l}_{p} \gamma_{\mu} l_{r}\right)\left(\bar{e}_{s} \gamma^{\mu} e_{t}\right)$ \\
$Q_{l u}$ & $\left(\bar{l}_{p} \gamma_{\mu} l_{r}\right)\left(\bar{u}_{s} \gamma^{\mu} u_{t}\right)$ \\
$Q_{l d}$ & $\left(\bar{l}_{p} \gamma_{\mu} l_{r}\right)\left(\bar{d}_{s} \gamma^{\mu} d_{t}\right)$ \\
$Q_{q e}$ & $\left(\bar{q}_{p} \gamma_{\mu} q_{r}\right)\left(\bar{e}_{s} \gamma^{\mu} e_{t}\right)$ \\
$Q_{q u}^{(1)}$ & $\left(\bar{q}_{p} \gamma_{\mu} q_{r}\right)\left(\bar{u}_{s} \gamma^{\mu} u_{t}\right)$ \\
$Q_{q u}^{(8)}$ & $\left(\bar{q}_{p} \gamma_{\mu} T^{A} q_{r}\right)\left(\bar{u}_{s} \gamma^{\mu} T^{A} u_{t}\right)$ \\
$Q_{q d}^{(1)}$ & $\left(\bar{q}_{p} \gamma_{\mu} q_{r}\right)\left(\bar{d}_{s} \gamma^{\mu} d_{t}\right)$ \\
$Q_{q d}^{(8)}$ & $\left(\bar{q}_{p} \gamma_{\mu} T^{A} q_{r}\right)\left(\bar{d}_{s} \gamma^{\mu} T^{A} d_{t}\right)$ \\
\hline
\end{tabular}

\begin{tabular}{|l|c|}
\hline \multicolumn{2}{|c|}{$8:(\bar{L} R)(\bar{L} R)+$ h.c. } \\
\hline$Q_{q u q d}^{(1)}$ & $\left(\bar{q}_{p}^{j} u_{r}\right) \epsilon_{j k}\left(\bar{q}_{s}^{k} d_{t}\right)$ \\
$Q_{q u q d}^{(8)}$ & $\left(\bar{q}_{p}^{j} T^{A} u_{r}\right) \epsilon_{j k}\left(\bar{q}_{s}^{k} T^{A} d_{t}\right)$ \\
$Q_{l e q u}^{(1)}$ & $\left(\bar{l}_{p}^{j} e_{r}\right) \epsilon_{j k}\left(\bar{q}_{s}^{k} u_{t}\right)$ \\
$Q_{l e q u}^{(3)}$ & $\left(\bar{l}_{p}^{j} \sigma_{\mu \nu} e_{r}\right) \epsilon_{j k}\left(\bar{q}_{s}^{k} \sigma^{\mu \nu} u_{t}\right)$ \\
\hline
\end{tabular}

Table 1. The 59 independent dimension-six operators built from Standard Model fields which conserve baryon number, as given in ref. [2]. The operators are divided into eight classes: $X^{3}, H^{6}$, etc. Operators with +h.c. in the table heading also have hermitian conjugates, as does the $\psi^{2} H^{2} D$ operator $Q_{H u d}$. The subscripts $p, r, s, t$ are flavor indices. 

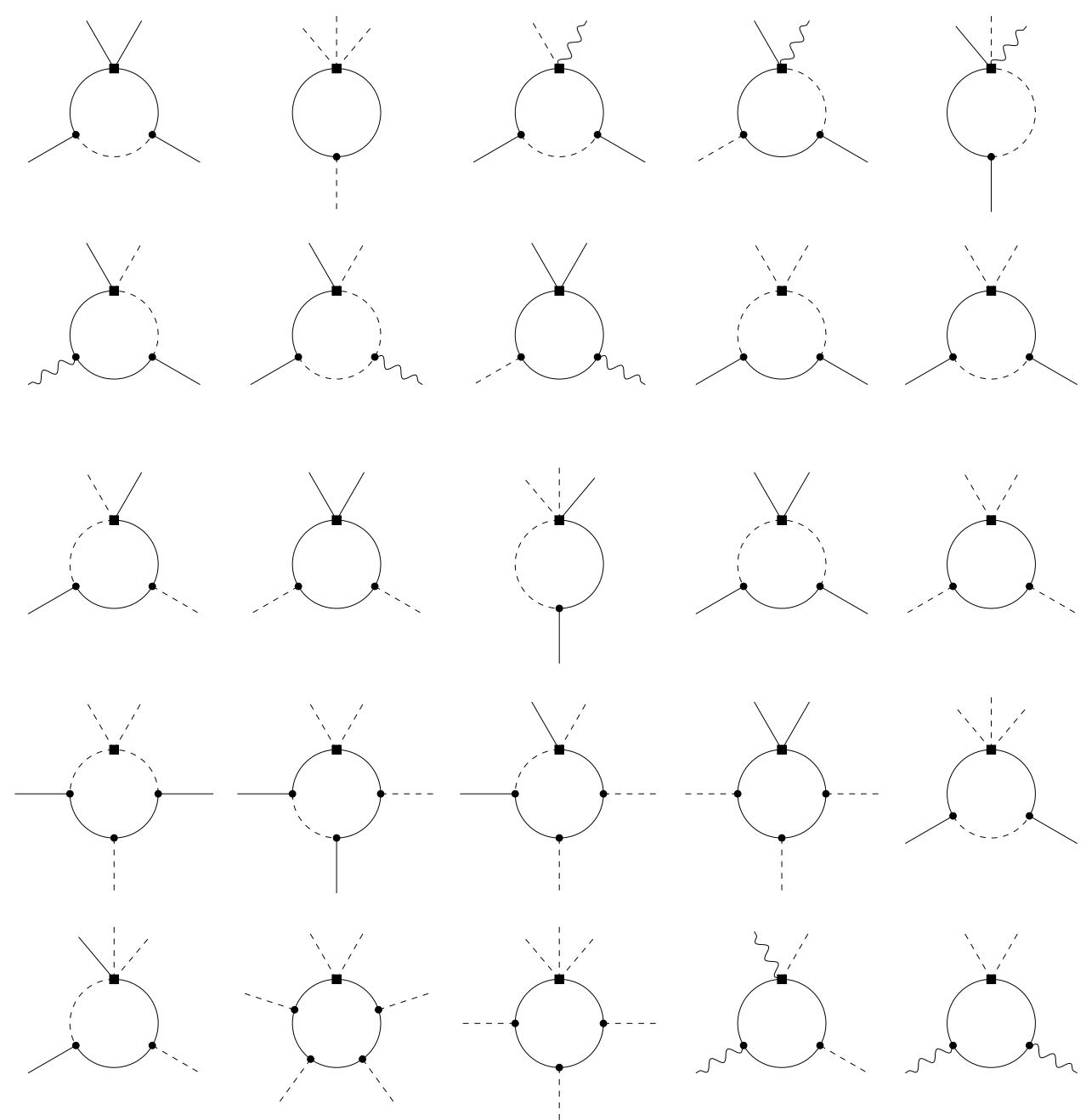

Figure 1. A selection of graphs contributing to the one-loop anomalous dimension matrix. The solid square is a $\mathcal{L}^{(6)}$ vertex, and the dots are SM vertices. Some graphs containing gauge fields contribute to the anomalous dimension matrix in the limit $g^{2} \rightarrow 0$ in the rescaled operator basis used for power counting. The first graph leads to anomalous dimension contributions seven pages long when all flavor combinations are evaluated.

where

$$
\begin{aligned}
\mathcal{L}_{\mathrm{SM}}= & -\frac{1}{4} G_{\mu \nu}^{A} G^{A \mu \nu}-\frac{1}{4} W_{\mu \nu}^{I} W^{I \mu \nu}-\frac{1}{4} B_{\mu \nu} B^{\mu \nu}+\left(D_{\mu} H^{\dagger}\right)\left(D^{\mu} H\right)+\sum_{\psi=q, u, d, l, e} \bar{\psi} i \not D \psi \\
& -\lambda\left(H^{\dagger} H-\frac{1}{2} v^{2}\right)^{2}-\left[H^{\dagger j} \bar{d} Y_{d} q_{j}+\widetilde{H}^{\dagger j} \bar{u} Y_{u} q_{j}+H^{\dagger j} \bar{e} Y_{e} l_{j}+\text { h.c. }\right],
\end{aligned}
$$

and $\mathcal{L}^{(d)}$ denotes terms in the effective Lagrangian of mass dimension $d$. In this work, we restrict our attention to the dimension-six Lagrangian

$$
\mathcal{L}^{(6)}=\sum_{i} C_{i} Q_{i},
$$


where the sum is over the 59 independent operators $Q_{i}$ given in table 1 . Each independent operator $Q_{i}$ appears in the sum with a corresponding operator coefficient $C_{i}$, which is proportional to $1 / \Lambda^{2}$.

The one-loop anomalous dimension matrix of the dimension-six operators has entries which are proportional to gauge couplings $g$, the Higgs scalar doublet self-coupling $\lambda$ and Yukawa couplings $y$. The coupling constant dependence of the anomalous dimension entries simplifies if the operators are normalized using naive dimensional analysis (NDA) [12], and a factor of $g$ is absorbed into each field-strength tensor $X$ and a factor of $y$ is absorbed into the single chirality-flip operators $\psi^{2} H^{3}$ and $\psi^{2} X H$. In terms of these rescaled operators $\widetilde{Q}_{i}$, the anomalous dimension entries have the form

$$
\gamma \propto\left(\frac{g^{2}}{16 \pi^{2}}\right)^{n_{g}}\left(\frac{\lambda}{16 \pi^{2}}\right)^{n_{\lambda}}\left(\frac{y^{2}}{16 \pi^{2}}\right)^{n_{y}}, \quad N=n_{g}+n_{\lambda}+n_{y}
$$

where $N$ is the perturbative order of the anomalous dimension. The form eq. (2.4), and a formula for $N$ in terms of NDA weights $w_{i}$ of the rescaled operators, was derived in ref. [13]. At one-loop order, $N=0,1,2,3,4$, so that entries in $\gamma$ range from perturbative order $N=0$, i.e. effectively "tree-level order," to perturbative order $N=4$, i.e. effectively "four-loop order." The form of the one-loop anomalous dimension matrix for the rescaled operators is given in table 1 of ref. [13].

The rescaled operators $\widetilde{Q}_{i}$ give the simple form eq. (2.4) for the coupling constant dependence of $\gamma$, which is useful to classify the terms in $\gamma$. However, the actual results given below are for the original unrescaled operator basis $Q_{i}$ of ref. [2]. Ref. [3] calculated the complete result for $\gamma_{44}$. Ref. [5] performed a complete classification of the entire anomalous dimension matrix, giving the allowed values of $n_{g}, n_{\lambda}, n_{y}$ for all possible terms. There are non-zero entries in $\gamma$ for which no one-particle irreducible graph exists, arising from operator conversions using the EOM. Ref. [5] calculated the order $\lambda, \lambda^{2}$ and $\lambda y^{2}$ terms of the one-loop anomalous dimension matrix, i.e. entries with $n_{\lambda} \geq 1$ and $n_{g}=0$. These anomalous dimensions had either $n_{y}=0$ or $n_{y}=1$. In this paper, we calculate the order $y^{2}$ and $y^{4}$ terms, which are the remaining terms with $n_{g}=0$. The terms with $n_{g} \neq 0$ will be discussed in a subsequent publication.

\section{Discussion}

The anomalous dimensions are lengthy, and are given in the appendix. Here, we briefly comment on some of the results.

The dimension-six operators alter the formulae of SM observables at tree level. For example, the Higgs doublet coupling to leptons in the SM EFT involves the Lagrangian terms

$$
\begin{aligned}
\mathcal{L} & =-\left[Y_{e}\right]_{r s} H^{\dagger j} \bar{e}_{r} l_{s j}+C_{e r}^{*}\left(H^{\dagger} H\right) H^{\dagger j} \bar{e}_{r} l_{s j}+h . c . \\
& =-\frac{1}{\sqrt{2}}(v+h) \bar{e}_{R r} Y_{e} e_{L s}+\frac{1}{2 \sqrt{2}}(v+h)^{3} C_{e H}^{*} \bar{e}_{R r} e_{L s}+h . c .
\end{aligned}
$$


where the second line has been evaluated in the spontaneously broken theory. Consequently, the charged lepton mass matrix

$$
\frac{1}{\sqrt{2}} v\left(\left[Y_{e}\right]_{r s}-\frac{1}{2} v^{2} C_{e H}^{*}\right)
$$

and the Higgs-charged lepton coupling matrix

$$
\frac{1}{\sqrt{2}}\left(\left[Y_{e}\right]_{r s}-\frac{3}{2} v^{2} C_{e r}^{*}\right)
$$

depend on different combinations of the usual SM $d=4$ Yukawa matrix $Y_{e}$ and the dimension-six terms $v^{2} C_{e H}^{*}$. The two combinations are not simultaneously diagonalizable in general, which can lead to flavor-changing Higgs couplings to fermions. Keeping only the top-Yukawa coupling contributions to the $C_{e H}$ anomalous dimension eq. (A.18),

$$
\underset{r s}{\dot{C}_{e H}}=\underset{r s t p}{4 C_{l e q u}^{(1)}} N_{c}\left[Y_{u} Y_{u}^{\dagger} Y_{u}\right]_{p t}+\ldots,
$$

where $\dot{C}$ is defined in eq. (A.1), we see that flavor violation from the four-fermion operator $Q_{\text {lequ }}^{(1)}=\left(\bar{l}_{p}^{j} e_{r}\right) \epsilon_{j k}\left(\bar{q}_{s}^{k} u_{t}\right)$ can feed into the lepton Yukawa couplings. Similar flavor-mixing terms are also present in the quark sector. These flavor-mixing effects, which depend on the flavor structure of the dimension-six operator coefficients, need not be suppressed by SM Yukawa couplings, and could be phenomenologically interesting. For some recent phenomenological studies of bounds on such Higgs related flavor violation, see refs. [14, 15].

The lepton dipole operators also get contributions from $\psi^{4}$ operators,

$$
\begin{gathered}
\dot{C}_{e W}=-2 g_{2} N_{c}\left(\mathrm{y}_{u}+\mathrm{y}_{q}\right) C_{l e q u}^{(3)}\left[Y_{u}\right]_{t p}+\ldots, \\
\dot{C}_{r s}=4 g_{1} N_{c}\left(\mathrm{y}_{u}+\mathrm{y}_{q}\right) C_{\text {lequ }}^{(3)}\left[Y_{u s p}\right]_{t p}+\ldots
\end{gathered}
$$

Such a mixing of "tree-generated" into "loop-generated" operators was recently argued to vanish in general, but it is non-zero by explicit computation. (See refs. [5, 16] for more discussion on related issues.) The linear combination

$$
\underset{r s}{\mathscr{C}_{e \gamma}}=\frac{1}{g_{1}} C_{e B}-\frac{1}{g_{2}} C_{e W}
$$

is the coefficient of the electromagnetic dipole operator

$$
\underset{r s}{e} \mathscr{C}_{e \gamma} F_{\mu \nu} \bar{l}_{r} \sigma^{\mu \nu} e_{s} H
$$

which gives flavor transitions such as $\mu \rightarrow e \gamma$, when $H$ is replaced by its vacuum expectation value $v / \sqrt{2}$. It has the anomalous dimension

$$
\underset{r s}{\dot{\mathscr{C}}_{\mathrm{r} \gamma}}=6 N_{c}\left(\mathrm{y}_{u}+\mathrm{y}_{q}\right) C_{\text {lequ }}^{(3)}\left[Y_{u}\right]_{t p}+\ldots
$$

The current bound on the $\mu \rightarrow e \gamma$ branching ratio from the MEG experiment [17] is $5.4 \times 10^{-13}$, with an order of magnitude improvement in sensitivity expected with a 
future MEG upgrade. As these sensitive probes of BSM flavor violation increase in precision, incorporating the RGE effects presented in this work will be crucial in correctly interpreting future limits or deviations from SM predictions. Furthermore, if deviations are not found, the non-trivial flavor mixing effects present in the RGEs puts strong constraints on the flavor structure of the SM EFT. One possible interpretation is that it implies the idea of a symmetry-based solution, such as minimal flavor violation [18-20], if BSM physics is not simply decoupled.

\section{Conclusion}

We have computed the Yukawa terms in the $59 \times 59$ one-loop anomalous dimension matrix of dimension-six operators in the Standard Model effective field theory. The anomalous dimensions mix the eight different operator classes of dimension-six operators, and the resultant expressions are lengthy. Most of the complications arise from the 25 four-fermion operators (class $\left.8: \psi^{4}\right)$. There are interesting non-trivial flavor effects which can occur from the flavor structure of the renormalization group evolution equations. Incorporating these RGE effects refines the interpretation of the increasingly strong bounds derived from the many searches for flavor violation beyond the SM.

\section{Acknowledgments}

We would like to thank R. Alonso for pointing out some corrections in the equations. This work was supported in part by DOE grant DE-SC0009919.

\section{A Results}

The renormalization group equations are given in this appendix. These equations are to be added to eqs. (4.3), (4.4), (6.1)-(6.4) of ref. [5]. Eq. (5.6) of ref. [5], which does not depend on the $\lambda\left(H^{\dagger} H\right)^{2}$ coupling, is included as part of the results of this paper in section A.6, and should not be added again. The invariants $c_{F, 3}=\left(N_{c}^{2}-1\right) /\left(2 N_{c}\right)$ and $c_{A, 3}=N_{c}$ are the $\mathrm{SU}(3)$ quadratic Casimirs in the fundamental and adjoint representation, respectively; $N_{c}=3$ is the number of colors, and $\mathrm{y}_{q, l, u, d, e}$ denotes the $\mathrm{U}(1)$ hypercharges of the fermions. We use the notation

$$
\dot{C} \equiv 16 \pi^{2} \mu \frac{\mathrm{d}}{\mathrm{d} \mu} C
$$

in the renormalization group equations given below. The wavefunction renormalization contributions proportional to Yukawa couplings are written in terms of

$$
\begin{aligned}
& \gamma_{l s}^{(Y)}=\frac{1}{2}\left[Y_{e}^{\dagger} Y_{e}\right]_{r s}, \quad \gamma_{r s}^{(Y)}=\left[Y_{e} Y_{e}^{\dagger}\right]_{r s}, \quad \gamma_{H}^{(Y)}=\operatorname{Tr}\left[N_{c} Y_{u}^{\dagger} Y_{u}+N_{c} Y_{d}^{\dagger} Y_{d}+Y_{e}^{\dagger} Y_{e}\right], \\
& \underset{r s}{\gamma_{q}^{(Y)}}=\frac{1}{2}\left[Y_{u}^{\dagger} Y_{u}+Y_{d}^{\dagger} Y_{d}\right]_{r s}, \quad \underset{r s}{\gamma_{u}^{(Y)}}=\left[Y_{u} Y_{u}^{\dagger}\right]_{r s}, \quad \underset{r s}{\gamma_{d}^{(Y)}}=\left[Y_{d} Y_{d}^{\dagger}\right]_{r s}
\end{aligned}
$$


which are $16 \pi^{2}$ times the field anomalous dimensions. The gauge contributions to wavefunction renormalization will be included with the gauge terms for the anomalous dimension matrix, since only the total combination is gauge invariant.

To simplify later expressions, it is useful to define the constants $\eta_{1-5}$ and $\xi_{e, d, u} \cdot \eta_{1,2}$ were already used in ref. [5].

$$
\begin{aligned}
& \eta_{1}=\frac{1}{2} N_{c} C_{d s}\left[Y_{d}\right]_{s r}+\frac{1}{2} N_{c} C_{d s}^{*}\left[Y_{d}^{\dagger}\right]_{r s}+\frac{1}{2} N_{c} C_{r H}\left[Y_{u}\right]_{s r}+\frac{1}{2} N_{c} C_{r s}^{*} \underset{r s}{*}\left[Y_{u}^{\dagger}\right]_{r s}+\frac{1}{2} C_{r s}\left[Y_{e}\right]_{s r}+\frac{1}{2} C_{r s}^{*}\left[Y_{e}^{\dagger}\right]_{r s}, \\
& \eta_{2}=-2 N_{c} C_{r q}^{(3)}\left[Y_{u}^{\dagger} Y_{u}+Y_{d}^{\dagger} Y_{d}\right]_{s r}+N_{c} C_{r u d}\left[Y_{d} Y_{u}^{\dagger}\right]_{s r}+N_{c} C_{H u d}^{*}\left[Y_{u} Y_{d}^{\dagger}\right]_{s r}-2 C_{r s}^{(3)}\left[Y_{e}^{\dagger} Y_{e}\right]_{s r}, \\
& \eta_{3}=N_{c} C_{r q}^{(1)}\left[Y_{d}^{\dagger} Y_{d}-Y_{u}^{\dagger} Y_{u}\right]_{s r}+3 N_{c} C_{r q}^{(3)}\left[Y_{d}^{\dagger} Y_{d}+Y_{u}^{\dagger} Y_{u}\right]_{s r}+N_{c} C_{r u}\left[Y_{u} Y_{u}^{\dagger}\right]_{s r}-N_{c} C_{r d}\left[Y_{d} Y_{d}^{\dagger}\right]_{s r} \\
& -N_{c} C_{H u d}\left[Y_{d} Y_{u}^{\dagger}\right]_{s r}-N_{c} C_{H u d}^{*}\left[Y_{u} Y_{d}^{\dagger}\right]_{s r}+\left(3 C_{r s}^{(3)}+C_{r s}^{(1)}\right)\left[Y_{e}^{\dagger} Y_{e}\right]_{s r}-C_{r s}\left[Y_{e} Y_{e}^{\dagger}\right]_{s r} \\
& \eta_{4}=4 N_{c} C_{r q}^{(1)}\left[Y_{d}^{\dagger} Y_{d}-Y_{u}^{\dagger} Y_{u}\right]_{s r}+4 N_{c} C_{r s}\left[Y_{u} Y_{u}^{\dagger}\right]_{s r}-4 N_{c} C_{r s}\left[Y_{d} Y_{d}^{\dagger}\right]_{s r}+\underset{r s}{2 N_{c} C_{H u d}}\left[Y_{d} Y_{u}^{\dagger}\right]_{s r} \\
& +2 N_{c} C_{H_{s r}}^{*}\left[Y_{u} Y_{d}^{\dagger}\right]_{s r}+4 C_{r s}^{(1)}\left[Y_{e}^{\dagger} Y_{e}\right]_{s r}-4 C_{r s}\left[Y_{e} Y_{e}^{\dagger}\right]_{s r} \\
& \eta_{5}=\frac{1}{2} i N_{c} C_{d H}\left[Y_{d s}\right]_{s r}-\frac{1}{2} i N_{c} C_{r s}^{*}\left[Y_{d}^{\dagger}\right]_{r s}-\frac{1}{2} i N_{c} C_{r s}\left[Y_{u}\right]_{s r}+\frac{1}{2} i N_{c} C_{r s}^{*}\left[Y_{u}^{\dagger}\right]_{r s}+\frac{1}{2} i C_{r s}^{e H}\left[Y_{e}\right]_{s r}-\frac{1}{2} i C_{r s}^{*}\left[Y_{e}^{\dagger}\right]_{r s}
\end{aligned}
$$

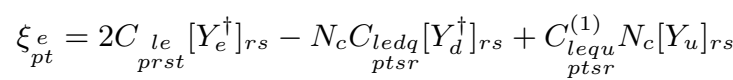

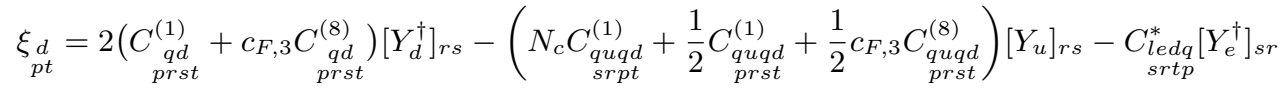

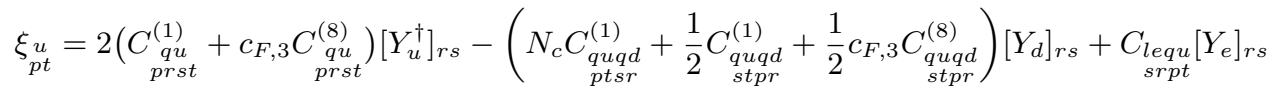

The Yukawa contributions to the one-loop renormalization group equations of the 59 dimension-six operator coefficients are listed by operator class in the following eight subsections.

\section{A.1 $X^{3}$}

$$
\dot{C}_{G}=0, \quad \dot{C}_{\widetilde{G}}=0, \quad \dot{C}_{W}=0, \quad \dot{C}_{\widetilde{W}}=0
$$

\section{A.2 $H^{6}$}

$$
\begin{aligned}
\dot{C}_{H}= & -4 N_{c} C_{r H}\left[Y_{u} Y_{u}^{\dagger} Y_{u}\right]_{s r}-4 N_{c} C_{r H}^{*}\left[Y_{u}^{\dagger} Y_{u} Y_{u}^{\dagger}\right]_{r s}-4 N_{c} C_{d H}\left[Y_{d} Y_{d}^{\dagger} Y_{d}\right]_{s r}-4 N_{c} C_{d H}^{*}\left[Y_{d}^{\dagger} Y_{d} Y_{d}^{\dagger}\right]_{r s} \\
& -4 C_{r s}\left[Y_{e} Y_{e}^{\dagger} Y_{e}\right]_{s r}-4 C_{e s}^{*}\left[Y_{e}^{\dagger} Y_{e} Y_{e}^{\dagger}\right]_{r s}+6 \gamma_{H}^{(Y)} C_{H}
\end{aligned}
$$

\section{A.3 $H^{4} D^{2}$}

$$
\dot{C}_{H \square}=-2 \eta_{3}+4 \gamma_{H}^{(Y)} C_{H \square} \quad \dot{C}_{H D}=-2 \eta_{4}+4 \gamma_{H}^{(Y)} C_{H D}
$$




\section{A.4 $X^{2} H^{2}$}

$$
\begin{aligned}
& \dot{C}_{H G}=-2 g_{3}\left(C_{r G}\left[Y_{u}\right]_{s r}+\left[Y_{u}^{\dagger}\right]_{r s} C_{r G}^{*}\right)-2 g_{3}\left(C_{d G}\left[Y_{r s}\right]_{s r}+\left[Y_{d}^{\dagger}\right]_{r s} C_{d G}^{*}\right)+2 \gamma_{H}^{(Y)} C_{H G} \\
& \dot{C}_{H \widetilde{G}}=2 g_{3}\left(i C_{r s}\left[Y_{u}\right]_{s r}-i\left[Y_{u}^{\dagger}\right]_{r s} C_{r s}^{*}\right)+2 g_{3}\left(i C_{d G}\left[Y_{d}\right]_{s r}-i\left[Y_{d}^{\dagger}\right]_{r s} C_{d s}^{*}\right)+2 \gamma_{H}^{(Y)} C_{H \widetilde{G}} \\
& \dot{C}_{H W}=-g_{2}\left(C_{e W}\left[Y_{e}\right]_{s r}+\left[Y_{e}^{\dagger}\right]_{r s} C_{r s}^{*}\right)-g_{2} N_{c}\left(C_{r W}\left[Y_{u s}\right]_{s r}+\left[Y_{u}^{\dagger}\right]_{r s} C_{r S}^{*}\right) \\
& -g_{2} N_{c}\left(C_{d W}\left[Y_{d}\right]_{s r}+\left[Y_{d}^{\dagger}\right]_{r s} C_{d W}^{*}\right)+2 \gamma_{H}^{(Y)} C_{H W} \\
& \dot{C}_{H \widetilde{W}}=g_{2}\left(i C_{e W}\left[Y_{e s}\right]_{s r}-i\left[Y_{e}^{\dagger}\right]_{r s} C_{e W}^{*}\right)+g_{2} N_{c}\left(i C_{r W}\left[Y_{u s}\right]_{s r}-i\left[Y_{u}^{\dagger}\right]_{r s} C_{r W}^{*}\right) \\
& +g_{2} N_{c}\left(i C_{d W}\left[Y_{d}\right]_{s r}-i\left[Y_{d}^{\dagger}\right]_{r s} C_{d W}^{*}\right)+2 \gamma_{H}^{(Y)} C_{H \widetilde{W}} \\
& \dot{C}_{H B}=-2 g_{1}\left(\mathrm{y}_{l}+\mathrm{y}_{e}\right)\left(C_{r s}\left[Y_{e}\right]_{s r}+\left[Y_{e}^{\dagger}\right]_{r s} C_{r s}^{*}\right)-2 g_{1} N_{c}\left(\mathrm{y}_{q}+\mathrm{y}_{u}\right)\left(C_{u B}\left[Y_{u}\right]_{s r}+\left[Y_{u}^{\dagger}\right]_{r s} C_{r s}^{*}\right) \\
& -2 g_{1} N_{c}\left(\mathrm{y}_{q}+\mathrm{y}_{d}\right)\left(C_{d B}\left[Y_{d s}\right]_{s r}+\left[Y_{d}^{\dagger}\right]_{r s} C_{r s}^{*}\right)+2 \gamma_{H}^{(Y)} C_{H B} \\
& \dot{C}_{H \widetilde{B}}=2 g_{1}\left(\mathrm{y}_{l}+\mathrm{y}_{e}\right)\left(i C_{r s}\left[Y_{e}\right]_{s r}-i\left[Y_{e}^{\dagger}\right]_{r s} C_{r s}^{*}\right)+2 g_{1} N_{c}\left(\mathrm{y}_{q}+\mathrm{y}_{u}\right)\left(i C_{r s}\left[Y_{u}\right]_{s r}-i\left[Y_{u}^{\dagger}\right]_{r s} C_{r s}^{*}\right) \\
& +2 g_{1} N_{c}\left(\mathrm{y}_{q}+\mathrm{y}_{d}\right)\left(i C_{d B}\left[Y_{d s}\right]_{s r}-i\left[Y_{d}^{\dagger}\right]_{r s} C_{d s}^{*}\right)+2 \gamma_{H}^{(Y)} C_{H \tilde{B}}
\end{aligned}
$$

$\dot{C}_{H W B}=-g_{2}\left(C_{e B}\left[Y_{e}\right]_{s r}+\left[Y_{e}^{\dagger}\right]_{r s} C_{e B}^{*}\right)+g_{2 s} N_{c}\left(C_{r s}\left[Y_{u}\right]_{s r}+\left[Y_{u}^{\dagger}\right]_{r s} C_{r s}^{*}\right)-g_{2} N_{c}\left(C_{d B}\left[Y_{r s}\right]_{s r}+\left[Y_{d}^{\dagger}\right]_{r s} C_{d B}^{*}\right)$$$
-2 g_{1}\left(\mathrm{y}_{l}+\mathrm{y}_{e}\right)\left(C_{e W}\left[Y_{e}\right]_{s r}+\left[Y_{e}^{\dagger}\right]_{r s} C_{e W}^{*}\right)+2 g_{1} N_{c}\left(\mathrm{y}_{q}+\mathrm{y}_{u}\right)\left(C_{r s}\left(Y_{u}\right]_{s r}+\left[Y_{u}^{\dagger}\right]_{r s} C_{r s}^{*}\right)
$$$$
-2 g_{1} N_{c}\left(\mathrm{y}_{q}+\mathrm{y}_{d}\right)\left(C_{d W}\left[Y_{d}\right]_{s r}+\left[Y_{d}^{\dagger}\right]_{r s} C_{d W}^{*}\right)+2 \gamma_{H}^{(Y)} C_{H W B}
$$

$\dot{C}_{H \widetilde{W} B}=g_{2}\left(i C_{e B}\left[Y_{e}\right]_{s r}-i\left[Y_{e}^{\dagger}\right]_{r s} C_{e B}^{*}\right)-g_{2} N_{c}\left(i C_{u B}\left[Y_{u}\right]_{s r}-i\left[Y_{u}^{\dagger}\right]_{r s} C_{u B}^{*}\right)+g_{2} N_{c}\left(i C_{d B}\left[Y_{d s}\right]_{s r}-i\left[Y_{d}^{\dagger}\right]_{r s} C_{d B}^{*}\right)$

$+2 g_{1}\left(\mathrm{y}_{l}+\mathrm{y}_{e}\right)\left(i C_{e W}\left[Y_{e}\right]_{s r}-i\left[Y_{e}^{\dagger}\right]_{r s} C_{e W}^{*}\right)-2 g_{1} N_{c}\left(\mathrm{y}_{q}+\mathrm{y}_{u}\right)\left(i C_{r s}\left[Y_{u}\right]_{s r}-i\left[Y_{u}^{\dagger}\right]_{r s} C_{r S}^{*}\right)$

$+2 g_{1} N_{c}\left(\mathrm{y}_{q}+\mathrm{y}_{d}\right)\left(i C_{d W}\left[Y_{d}\right]_{s r}-i\left[Y_{d}^{\dagger}\right]_{r s} C_{d W}^{*}\right)+2 \gamma_{H}^{(Y)} C_{H \widetilde{W} B}$

\section{A.5 $\psi^{2} H^{3}$}

$$
\begin{aligned}
& \dot{C}_{r s}^{\dot{u}_{r}}=2\left(\eta_{1}+\eta_{2}-i \eta_{5}\right)\left[Y_{u}^{\dagger}\right]_{r s}+\left[Y_{u}^{\dagger} Y_{u} Y_{u}^{\dagger}\right]_{r s}\left(C_{H D}-6 C_{H \square}\right)-\underset{r t}{2 C_{H q}^{(1)}}\left[Y_{u}^{\dagger} Y_{u} Y_{u}^{\dagger}\right]_{t s}+\underset{r t}{6 C_{H q}^{(3)}}\left[Y_{d}^{\dagger} Y_{d} Y_{u}^{\dagger}\right]_{t s}
\end{aligned}
$$

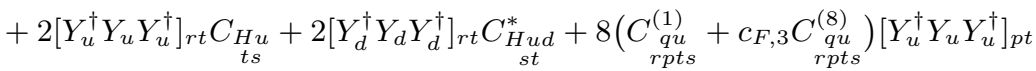

$$
\begin{aligned}
& -2\left(2 N_{c} C_{r u q d}^{(1)}+C_{r s t p}^{(1)}+c_{t s r p}^{(1)}+c_{F, 3} C_{\text {quqd }}^{(8)}\right)\left[Y_{d} Y_{d}^{\dagger} Y_{d}\right]_{p t}+4 C_{\text {lequ }}^{(1)}\left[Y_{\text {tprs }}^{\dagger} Y_{e} Y_{e}^{\dagger}\right]_{p t}+4 C_{u H}\left[Y_{u} Y_{u}^{\dagger}\right]_{t s}+5\left[Y_{u}^{\dagger} Y_{u}\right]_{r t} C_{u H} \\
& -2\left[Y_{d}^{\dagger}\right]_{r t} C_{d H}^{*}\left[Y_{u t}^{\dagger}\right]_{u s}-C_{d H}\left[Y_{d} Y_{u}^{\dagger}\right]_{t s}-2\left[Y_{d}^{\dagger} Y_{d}\right]_{r t} C_{u H}+3 \gamma_{t s}^{(Y)} C_{\substack{u s \\
r s}}+\underset{r v}{(Y)} C_{v H}+\underset{v v}{C_{u H}} \gamma_{v s}^{(Y)} \\
& \dot{C}_{r s}=2\left(\eta_{1}+\eta_{2}+i \eta_{5}\right)\left[Y_{d}^{\dagger}\right]_{r s}+\left[Y_{d}^{\dagger} Y_{d} Y_{d}^{\dagger}\right]_{r s}\left(C_{H D}-6 C_{H \square}\right)+2 C_{r q}^{(1)}\left[Y_{d}^{\dagger} Y_{d} Y_{d}^{\dagger}\right]_{t s}+6 C_{r t}^{(3)}\left[Y_{u}^{\dagger} Y_{u} Y_{d}^{\dagger}\right]_{t s} \\
& -2\left[Y_{d}^{\dagger} Y_{d} Y_{d}^{\dagger}\right]_{r t} C_{t s} C_{t s}-2\left[Y_{u}^{\dagger} Y_{u} Y_{u}^{\dagger}\right]_{r t} C_{H_{t s} u d}+8\left(C_{\substack{q d \\
r p t s}}^{(1)}+c_{F, 3} C_{\substack{q d \\
r p t s}}^{(8)}\right)\left[Y_{d}^{\dagger} Y_{d} Y_{d}^{\dagger}\right]_{p t}-4 C_{l e d q}^{*}\left[Y_{e}^{\dagger} Y_{e} Y_{e}^{\dagger}\right]_{p t} \\
& -2\left(2 N_{c} C_{\substack{q u q d \\
t p r s}}^{(1)}+C_{\substack{q u q d \\
\text { tppts }}}^{(1)}+c_{F, 3} C_{\substack{q u q d \\
r p t s}}^{(8)}\right)\left[Y_{u} Y_{u}^{\dagger} Y_{u}\right]_{p t}+4 C_{d H}\left[Y_{d} Y_{d}^{\dagger}\right]_{t s}+5\left[Y_{d}^{\dagger} Y_{d}\right]_{r t} C_{d H}{ }_{t s} \\
& +2\left[Y_{u}^{\dagger}\right]_{r t} C_{u t}^{*}\left[Y_{d}^{\dagger}\right]_{u s}-C_{u t}\left[Y_{u} Y_{d}^{\dagger}\right]_{t s}-2\left[Y_{u}^{\dagger} Y_{u}\right]_{r t} C_{d s}+3 \gamma_{H}^{(Y)} C_{d H}+\gamma_{r v}^{(Y)} C_{d H}+C_{r s} C_{r v} \gamma_{v s}^{(Y)} \\
& \dot{C}_{e H}=2\left(\eta_{1}+\eta_{2}+i \eta_{5}\right)\left[Y_{e}^{\dagger}\right]_{r s}+\left[Y_{e}^{\dagger} Y_{e} Y_{e}^{\dagger}\right]_{r s}\left(C_{H D}-6 C_{H \square}\right)+2 C_{r t}^{(1)}\left[Y_{e}^{\dagger} Y_{e} Y_{e}^{\dagger}\right]_{t s}-2\left[Y_{e}^{\dagger} Y_{e} Y_{e}^{\dagger}\right]_{r t} C_{H e} \\
& +8 C_{r p t s}^{l e}\left[Y_{e}^{\dagger} Y_{e} Y_{e}^{\dagger}\right]_{p t}-4 C_{l e d q} N_{c}\left[Y_{d}^{\dagger} Y_{d} Y_{d}^{\dagger}\right]_{t p}+4 C_{l e q u}^{(1)} N_{c}\left[Y_{u} Y_{u}^{\dagger} Y_{u}\right]_{p t}+4 C_{e H}\left[Y_{e} Y_{e}^{\dagger}\right]_{t s}
\end{aligned}
$$

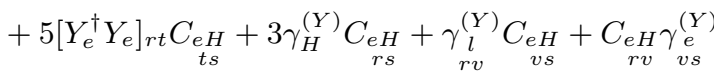




\section{A. $6 \psi^{2} X H$}

$$
\begin{aligned}
& \dot{C}_{e W}=-2 g_{2} N_{c}\left(\mathrm{y}_{u}+\mathrm{y}_{q}\right) C_{r s q u}^{(3)}\left[Y_{u}\right]_{t p}+\underset{r t}{C_{e W}}\left[Y_{e} Y_{e}^{\dagger}\right]_{t s}+\gamma_{H}^{(Y)}{\underset{r s}{e W}}_{r s}+\underset{r v}{\gamma_{l}^{(Y)}} C_{e W}+\underset{v s}{C_{e W} \gamma_{e s}^{(Y)}}
\end{aligned}
$$

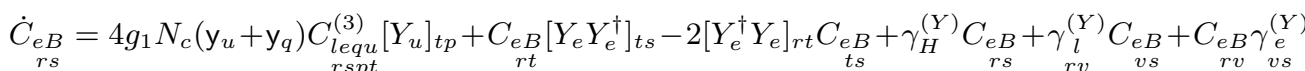

$$
\begin{aligned}
& \dot{C}_{r G}=-2\left[Y_{u}^{\dagger} Y_{u}+Y_{d}^{\dagger} Y_{d}\right]_{r t} C_{u G}-C_{d s}\left[Y_{r t} Y_{u}^{\dagger}\right]_{t s}+C_{r t} C_{r G}\left[Y_{u} Y_{u}^{\dagger}\right]_{t s}+\gamma_{H}^{(Y)} C_{r s}+\underset{r v}{\gamma_{r}} C_{v s}^{(Y)} C_{v G}+\underset{r v}{C_{u G}} \gamma_{v s}^{(Y)} \\
& \dot{C}_{u W}=-2 g_{2}\left(\mathrm{y}_{e}+\mathrm{y}_{l}\right) C_{\text {lequ }}^{(3)}\left[Y_{e}\right]_{p t}+2\left[Y_{d}^{\dagger} Y_{d}\right]_{r t} C_{u W}-C_{d w}\left[Y_{d} Y_{u}^{\dagger}\right]_{t s}+C_{r t}\left[Y_{u} Y_{u}^{\dagger}\right]_{t s} \\
& +\gamma_{H}^{(Y)} C_{u W}+\underset{r v}{\gamma_{r}^{(Y)} C_{u W}}+\underset{v v}{C_{u W}} \gamma_{v s}^{(Y)}
\end{aligned}
$$

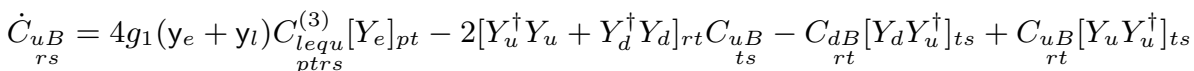

$$
\begin{aligned}
& +\gamma_{H}^{(Y)} C_{r s}+\gamma_{r v}^{(Y)} C_{u B}+C_{v s} C_{r v} \gamma_{v s}^{(Y)} \\
& \dot{C}_{d G}=-2\left[Y_{u}^{\dagger} Y_{u}+Y_{d}^{\dagger} Y_{d}\right]_{r t} C_{d G}-C_{r t}\left[Y_{u} Y_{d}^{\dagger}\right]_{t s}+C_{r t} C_{r G}\left[Y_{d} Y_{d}^{\dagger}\right]_{t s}+\gamma_{H}^{(Y)} C_{d G}+\underset{r v}{\gamma_{r}^{(Y)}} C_{d G}+C_{d G} C_{r v} \gamma_{d s}^{(Y)} \\
& \dot{C}_{d W}=2\left[Y_{u}^{\dagger} Y_{u}\right]_{r t} C_{d W}-C_{t s}\left[\begin{array}{c}
r t \\
r t
\end{array} Y_{u} Y_{d}^{\dagger}\right]_{t s}+C_{d W}\left[Y_{d} Y_{d}^{\dagger}\right]_{t s}+\gamma_{H}^{(Y)} C_{d W}+\gamma_{r v}^{(Y)} C_{d W}+C_{v s} \gamma_{r v} \gamma_{d s}^{(Y)}
\end{aligned}
$$

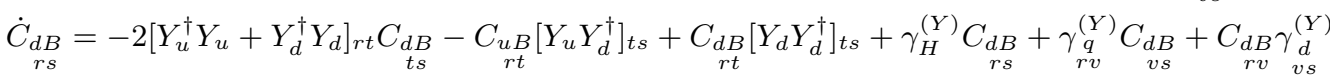

\section{A.7 $\psi^{2} H^{2} D$}

$$
\begin{aligned}
& \dot{C}_{\text {pr }}^{(1)}=\frac{1}{2}\left[Y_{u}^{\dagger} Y_{u}-Y_{d}^{\dagger} Y_{d}\right]_{p r}\left(C_{H \square}+C_{H D}\right)-\left[Y_{u}^{\dagger}\right]_{p s} C_{s t} C_{s u}\left[Y_{u}\right]_{t r}-\left[Y_{d}^{\dagger}\right]_{p s} C_{s t}\left[\begin{array}{rl}
H d \\
s t
\end{array}\right]_{t r}+2\left(C_{p r s t}^{q e}-C_{s t p r}^{(1)}\right)\left[Y_{e}^{\dagger} Y_{e}\right]_{t s} \\
& +\frac{3}{2}\left[Y_{d}^{\dagger} Y_{d}+Y_{u}^{\dagger} Y_{u}\right]_{p t} C_{t r}^{(1)}+\frac{3}{2} C_{p q}^{(1)}\left[Y_{d}^{\dagger} Y_{d}+Y_{u}^{\dagger} Y_{u}\right]_{t r}+\frac{9}{2}\left[Y_{d}^{\dagger} Y_{d}-Y_{u}^{\dagger} Y_{u}\right]_{p t} C_{t r}^{(3)}+\frac{9}{2} C_{H q}^{(3)}\left[Y_{d}^{\dagger} Y_{d}-Y_{u}^{\dagger} Y_{u}\right]_{t r}
\end{aligned}
$$

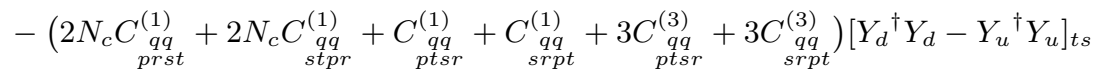

$$
\begin{aligned}
& -2 N_{c} C_{p r s t}^{(1)}\left[Y_{u} Y_{u}^{\dagger}\right]_{t s}+2 N_{c} C_{p d}^{(1)}\left[Y_{d} Y_{d}^{\dagger}\right]_{t s}+2 \gamma_{H}^{(Y)} C_{\substack{H q \\
p r}}^{(1)}+\gamma_{p t}^{(Y)} C_{t r}^{(1)}+\underset{\text { tr }}{(1)}+\underset{p t}{(1)} \gamma_{t r}^{(Y)}
\end{aligned}
$$

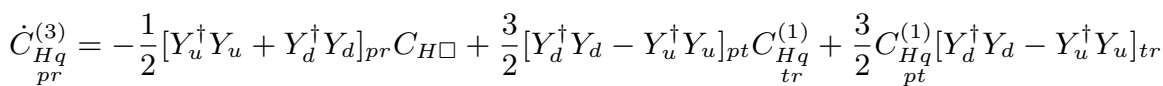

$$
\begin{aligned}
& +\frac{1}{2}\left[Y_{d}^{\dagger} Y_{d}+Y_{u}^{\dagger} Y_{u}\right]_{p t} C_{t r}^{(3)}+\frac{1}{2} C_{t q}^{(3)}\left[Y_{d}^{\dagger} Y_{d}+Y_{u}^{\dagger} Y_{u}\right]_{t r}
\end{aligned}
$$

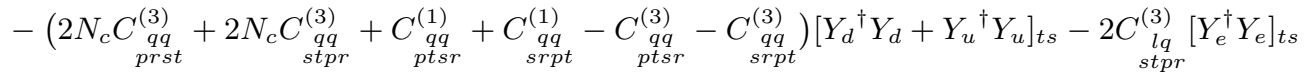

$$
\begin{aligned}
& +2 \gamma_{H}^{(Y)} C_{p q}^{(3)}+\underset{p t}{\gamma_{q}^{(Y)}} C_{t r}^{(3)}+\underset{p q}{C_{t r}^{(3)}} \gamma_{t r}^{(Y)} \\
& \left.\dot{C}_{H_{p r}}=\left[Y_{d} Y_{d}^{\dagger}\right]_{p r}\left(C_{H \square}+C_{H D}\right)-2\left[Y_{d}\right]_{p s} C_{H_{s t}}^{(1)}\left[Y_{d}^{\dagger}\right]_{t r}+3\left[Y_{d} Y_{d}^{\dagger}\right]_{p t} C_{t r}+3 C_{p t} d Y_{d} Y_{d}^{\dagger}\right]_{t r}-\left[Y_{d} Y_{u}^{\dagger}\right]_{p t} C_{H_{t r} u d} \\
& -C_{t p u d}^{*}\left[Y_{u} Y_{d}^{\dagger}\right]_{t r}+2\left(N_{c} C_{p r s t}^{d d}+N_{c} C_{s t p r}^{d d}+C_{p t s r}^{d d}+C_{s r p t}^{d d}\right)\left[Y_{d} Y_{d}^{\dagger}\right]_{t s}+2\left(C_{s t p r}^{e d}-C_{s t p r}\right)\left[Y_{e} Y_{e}^{\dagger}\right]_{t s} \\
& -2 N_{c} C_{\substack{u d \\
\text { stpr }}}^{(1)}\left[Y_{u} Y_{u}^{\dagger}\right]_{t s}-2 N_{c} C_{\substack{q d \\
\text { stpr }}}^{(1)}\left[Y_{d}^{\dagger} Y_{d}-Y_{u}^{\dagger} Y_{u}\right]_{t s}+2 \gamma_{H}^{(Y)} C_{p r}^{H d}+\gamma_{p t}^{(Y)} C_{t r}{ }_{t r}+\underset{p t}{C_{t r} \gamma_{t r}^{(Y)}} \\
& \dot{C}_{p u}=-\left[Y_{u} Y_{u}^{\dagger}\right]_{p r}\left(C_{H \square}+C_{H D}\right)-2\left[Y_{u}\right]_{p s} C_{H t}^{(1)}\left[Y_{u}^{\dagger}\right]_{t r}+3\left[Y_{u} Y_{u}^{\dagger}\right]_{p t} C_{t r}+\underset{p t}{3 C_{H u}}\left[Y_{u} Y_{u}^{\dagger}\right]_{t r}+\left[Y_{u} Y_{d}^{\dagger}\right]_{p t} C_{H t}^{*}{ }_{r t}^{*} \\
& +C_{p t}^{H u d}\left[Y_{d} Y_{u}^{\dagger}\right]_{t r}-2\left(N_{c} C_{p r s t}^{u u}+N_{c} C_{s t p r}^{u u}+C_{p t s r}^{u u}+C_{s r p t}^{u u}\right)\left[Y_{u} Y_{u}^{\dagger}\right]_{t s}+2\left(C_{s t p r}^{e u}-C_{s t p r}^{l u}\right)\left[Y_{e} Y_{e}^{\dagger}\right]_{t s} \\
& +2 N_{c} C_{\substack{u d \\
\text { prst }}}^{(1)}\left[Y_{d} Y_{d}^{\dagger}\right]_{t s}-2 N_{c} C_{\text {stpr }}^{(1)}\left[Y_{d}^{\dagger} Y_{d}-Y_{u}^{\dagger} Y_{u}\right]_{t s}+2 \gamma_{H}^{(Y)} C_{p r}+\gamma_{p t}^{(Y)} C_{t r}+\underset{p t}{C_{p u} \gamma_{t r}^{(Y)}} \\
& \dot{C}_{p u d}=\left[Y_{u} Y_{d}^{\dagger}\right]_{p r}\left(2 C_{H \square}-C_{H D}\right)-2\left[Y_{u} Y_{d}^{\dagger}\right]_{p t} C_{t r} C_{t r}+6 C_{p t} C_{p u}\left[Y_{u} Y_{d}^{\dagger}\right]_{t r}+2\left(C_{p t s r}^{(1)}+c_{F, 3} C_{\substack{u d \\
p t s r}}^{(8)}\right)\left[Y_{u} Y_{d}^{\dagger}\right]_{t s}
\end{aligned}
$$

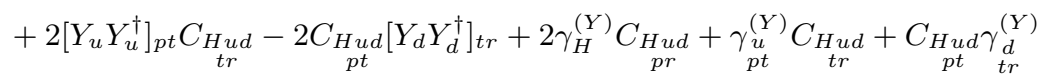




$$
\begin{aligned}
& \dot{C}_{p l}^{(1)}=-\frac{1}{2}\left[Y_{e}^{\dagger} Y_{e}\right]_{p r}\left(C_{H \square}+C_{H D}\right)-\left[Y_{e}^{\dagger}\right]_{p s} C_{s t}\left[Y_{e}\right]_{t r}+\frac{3}{2}\left[Y_{e}^{\dagger} Y_{e}\right]_{p t}\left(C_{t r}^{(1)}+3 C_{t r}^{(3)}\right)+\frac{3}{2}\left(C_{p t}^{(1)}+3 C_{p l}^{(3)}\right)\left[Y_{e}^{\dagger} Y_{e}\right]_{t r} \\
& +\left(2 C_{p r s t}^{l e}-2 C_{p r s t}^{l l}-2 C_{s t p r}^{u l}-C_{p t s r}^{u l}-C_{s r p t}^{l l}\right)\left[Y_{e}^{\dagger} Y_{e}\right]_{t s}-2 N_{c} C_{\text {prst }}^{(1)}\left[Y_{d}^{\dagger} Y_{d}-Y_{u}^{\dagger} Y_{u}\right]_{t s} \\
& -2 N_{c} C_{p r s t}^{l u}\left[Y_{u} Y_{u}^{\dagger}\right]_{t s}+2 N_{c} C_{p r s t}\left[Y_{d} Y_{d}^{\dagger}\right]_{t s}+2 \gamma_{H}^{(Y)} C_{p r}^{(1)}+\gamma_{p t}^{(Y)} C_{t r}^{(1)}+\underset{p t}{C_{t r}^{(1)}} \gamma_{t r}^{(Y)} \\
& \dot{C}_{p l}^{(3)}=-\frac{1}{2}\left[Y_{e}^{\dagger} Y_{e}\right]_{p r} C_{H \square}+\frac{1}{2}\left[Y_{e}^{\dagger} Y_{e}\right]_{p t}\left(3 C_{t r}^{(1)}+C_{t r}^{(3)}\right)+\frac{1}{2}\left(3 C_{p t}^{(1)}+C_{p t}^{(3)}\right)\left[Y_{e}^{\dagger} Y_{e}\right]_{t r} \\
& -\left(\underset{p t s r}{l l}+C_{s r p t}^{l l}\right)\left[Y_{e}^{\dagger} Y_{e}\right]_{t s}-2 N_{c} C_{p r s t}^{(3)}\left[Y_{d}^{\dagger} Y_{d}+Y_{u}^{\dagger} Y_{u}\right]_{t s}+2 \gamma_{H}^{(Y)} C_{p r}^{(3)}+\gamma_{p t}^{(Y)} C_{t r}^{(3)}+C_{p t}^{(3)} \gamma_{t r}^{(Y)} \\
& \dot{C}_{p r}=\left[Y_{e} Y_{e}^{\dagger}\right]_{p r}\left(C_{H \square}+C_{H D}\right)-2\left[Y_{e}\right]_{p s} C_{s t}^{(1)}\left[Y_{e}^{\dagger}\right]_{t r}+3\left[Y_{e} Y_{e}^{\dagger}\right]_{p t} C_{t r}+\underset{p t}{3 C_{H e}}\left[Y_{e} Y_{e}^{\dagger}\right]_{t r}
\end{aligned}
$$

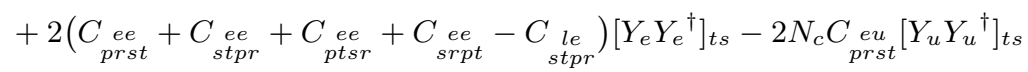

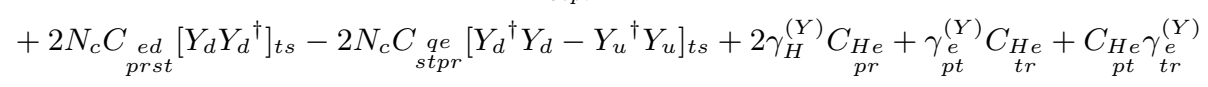

\section{A. $8 \psi^{4}$}

\section{A.8.1 $(\bar{L} L)(\bar{L} L)$}

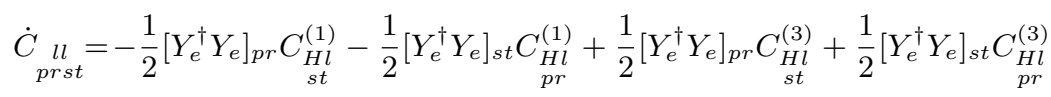

$$
\begin{aligned}
& -\left[Y_{e}^{\dagger} Y_{e}\right]_{s r} C_{p l}^{(3)}-\left[Y_{e}^{\dagger} Y_{e}\right]_{p t} C_{H r}^{(3)}-\frac{1}{2}\left[Y_{e}^{\dagger}\right]_{s v}\left[Y_{e}\right]_{w t} C_{p r v w}^{l e}-\frac{1}{2}\left[Y_{e}^{\dagger}\right]_{p v}\left[Y_{e}\right]_{w r} C_{s t v w}^{l e} \\
& +\underset{p v}{\gamma_{l}^{(Y)}} C_{v r s t}^{u l}+\gamma_{s v}^{(Y)} C_{p r v t}^{l l}+C_{p v s t}^{u l} \underset{v r}{\gamma_{l}^{(Y)}}+C_{p r s v}^{l l} \underset{v t}{\gamma_{l}^{(Y)}}
\end{aligned}
$$

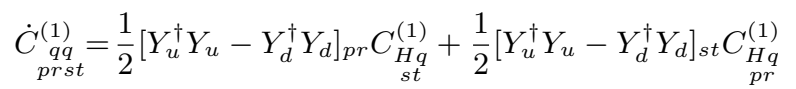

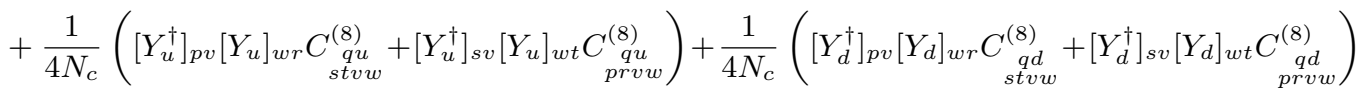

$$
\begin{aligned}
& -\frac{1}{8}\left(\left[Y_{u}^{\dagger}\right]_{p v}\left[Y_{u}\right]_{w t} C_{\substack{q u \\
\text { srvw }}}^{(8)}+\left[Y_{u}^{\dagger}\right]_{s v}\left[Y_{u}\right]_{w r} C_{\substack{q u \\
p t v w}}^{(8)}\right)-\frac{1}{8}\left(\left[Y_{d}^{\dagger}\right]_{p v}\left[Y_{d}\right]_{w t} C_{\substack{q d \\
\text { stvw }}}^{(8)}+\left[Y_{d}^{\dagger}\right]_{s v}\left[Y_{d}\right]_{w r} C_{\substack{q d \\
p t v w}}^{(8)}\right)
\end{aligned}
$$

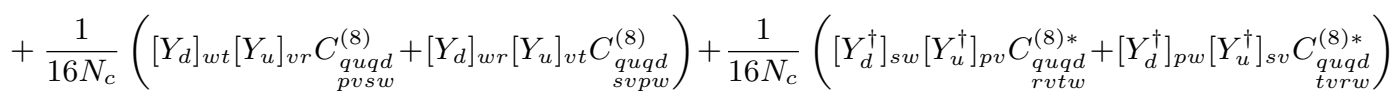

$$
\begin{aligned}
& +\frac{1}{16}\left(\left[Y_{d}\right]_{w t}\left[Y_{u}\right]_{v r} C_{\substack{q u q d \\
\text { svpw }}}^{(8)}+\left[Y_{d}\right]_{w r}\left[Y_{u}\right]_{v t} C_{\substack{q u q d \\
\text { pvsw }}}^{(8)}\right)+\frac{1}{16}\left(\left[Y_{d}^{\dagger}\right]_{s w}\left[Y_{u}^{\dagger}\right]_{p v} C_{\substack{q u q d \\
\text { turw }}}^{(8) *}+\left[Y_{d}^{\dagger}\right]_{p w}\left[Y_{u}^{\dagger}\right]_{s v} C_{\substack{q u q d \\
\text { rvtw }}}^{(8) *}\right) \\
& -\frac{1}{2}\left[Y_{u}^{\dagger}\right]_{p v}\left[Y_{u}\right]_{w r} C_{\substack{q u \\
\text { stvw }}}^{(1)}-\frac{1}{2}\left[Y_{d}^{\dagger}\right]_{p v}\left[Y_{d}\right]_{w r} C_{\substack{q d \\
s t v w}}^{(1)}-\frac{1}{2}\left[Y_{u}^{\dagger}\right]_{s v}\left[Y_{u}\right]_{w t} C_{\substack{q u \\
\text { prvw }}}^{(1)}-\frac{1}{2}\left[Y_{d}^{\dagger}\right]_{s v}\left[Y_{d}\right]_{w t} C_{\substack{q d \\
\text { prvw }}}^{(1)} \\
& -\frac{1}{8}\left[Y_{d}\right]_{w t}\left[Y_{u}\right]_{v r} C_{\substack{q u q d \\
\text { pvsw }}}^{(1)}-\frac{1}{8}\left[Y_{d}^{\dagger}\right]_{s w}\left[Y_{u}^{\dagger}\right]_{p v} C_{\substack{q u q d \\
\text { rvtw }}}^{(1) *}-\frac{1}{8}\left[Y_{d}\right]_{w r}\left[Y_{u}\right]_{v t} C_{\substack{\text { qud } \\
\text { svpw }}}^{(1)}-\frac{1}{8}\left[Y_{d}^{\dagger}\right]_{p w}\left[Y_{u}^{\dagger}\right]_{s v} C_{\substack{\text { qud } \\
\text { tvrw }}}^{(1) *}
\end{aligned}
$$

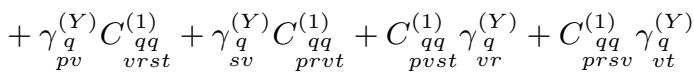

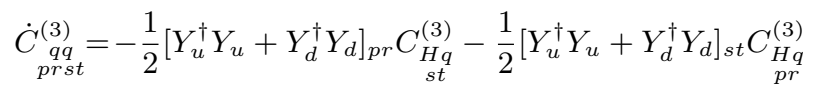

$$
\begin{aligned}
& -\frac{1}{8}\left(\left[Y_{u}^{\dagger}\right]_{p v}\left[Y_{u}\right]_{w t} C_{\substack{q u \\
\text { srvw }}}^{(8)}+\left[Y_{u}^{\dagger}\right]_{s v}\left[Y_{u}\right]_{w r} C_{\substack{q u \\
p t v w}}^{(8)}\right)-\frac{1}{8}\left(\left[Y_{d}^{\dagger}\right]_{p v}\left[Y_{d}\right]_{w t} C_{\substack{q d \\
\text { srvw }}}^{(8)}+\left[Y_{d}^{\dagger}\right]_{s v}\left[Y_{d}\right]_{w r} C_{\substack{q d \\
p t v w}}^{(8)}\right)
\end{aligned}
$$

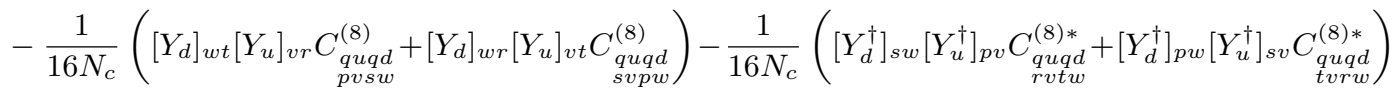

$$
\begin{aligned}
& -\frac{1}{16}\left(\left[Y_{d}\right]_{w t}\left[Y_{u}\right]_{v r} C_{\substack{q u q d \\
\text { svpw }}}^{(8)}+\left[Y_{d}\right]_{w r}\left[Y_{u}\right]_{v t} C_{\substack{q u q d \\
\text { pusw }}}^{(8)}\right)-\frac{1}{16}\left(\left[Y_{d}^{\dagger}\right]_{s w}\left[Y_{u}^{\dagger}\right]_{p v} C_{\substack{q u q d \\
\text { turw }}}^{(8) *}+\left[Y_{d}^{\dagger}\right]_{p w}\left[Y_{u}^{\dagger}\right]_{s v} C_{\substack{q u q d \\
\text { rvtw }}}^{(8) *}\right) \\
& +\frac{1}{8}\left[Y_{d}\right]_{w t}\left[Y_{u}\right]_{v r} C_{\substack{q u q d \\
\text { pusw }}}^{(1)}+\frac{1}{8}\left[Y_{d}^{\dagger}\right]_{s w}\left[Y_{u}^{\dagger}\right]_{p v} C_{\substack{q u q d \\
\text { rvtw }}}^{(1) *}+\frac{1}{8}\left[Y_{d}\right]_{w r}\left[Y_{u}\right]_{v t} C_{\substack{q \text { qud } \\
\text { svp }}}^{(1)}+\frac{1}{8}\left[Y_{d}^{\dagger}\right]_{p w}\left[Y_{u}^{\dagger}\right]_{s v} C_{\substack{q u q d \\
\text { tvrw }}}^{(1) *}
\end{aligned}
$$




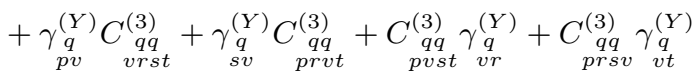

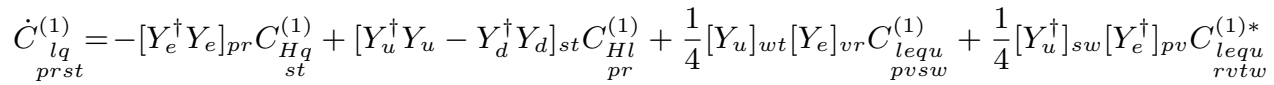

$$
\begin{aligned}
& -\left[Y_{u}^{\dagger}\right]_{s v}\left[Y_{u}\right]_{w t} C_{p r v w}^{l u}-\left[Y_{d}^{\dagger}\right]_{s v}\left[Y_{d}\right]_{w t} C_{\text {prvw }}^{l d}-\left[Y_{e}^{\dagger}\right]_{p v}\left[Y_{e}\right]_{w r} C_{s t v w}^{q e}
\end{aligned}
$$

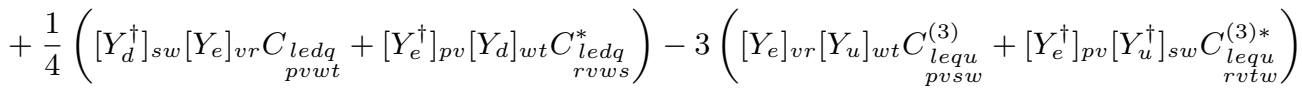

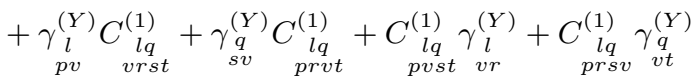

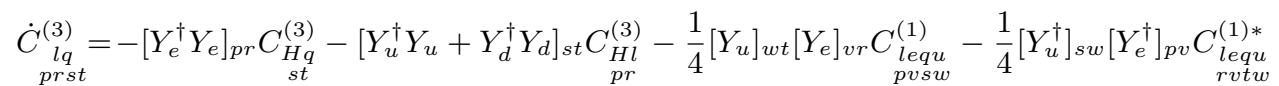

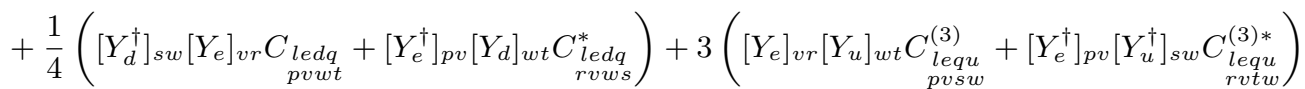

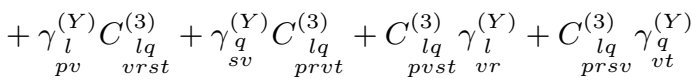

\section{A.8.2 $(\bar{R} R)(\bar{R} R)$}

$$
\begin{aligned}
& \dot{C}_{p e e}=\left[Y_{e} Y_{e}^{\dagger}\right]_{p r} C_{{ }_{s t}}+\left[Y_{e} Y_{e}^{\dagger}\right]_{s t} C_{p r}-\left[Y_{e}^{\dagger}\right]_{w r}\left[Y_{e}\right]_{p v} C_{v w s t}^{l e}-\left[Y_{e}^{\dagger}\right]_{w t}\left[Y_{e}\right]_{s v} C_{v w p r}^{l e}
\end{aligned}
$$

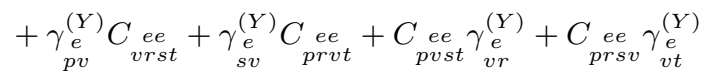

$$
\begin{aligned}
& \dot{C}_{p r s t}^{u u}=-\left[Y_{u} Y_{u}^{\dagger}\right]_{p r} C_{s t} C_{s t}-\left[Y_{u} Y_{u}^{\dagger}\right]_{s t} C_{p u}-\left[Y_{u}^{\dagger}\right]_{w r}\left[Y_{u}\right]_{p v} C_{v w s t}^{(1)}-\left[Y_{u}^{\dagger}\right]_{w t}\left[Y_{u}\right]_{s v} C_{v w p r}^{(1)} \\
& +\frac{1}{2 N_{c}}\left[Y_{u}\right]_{p v}\left[Y_{u}^{\dagger}\right]_{w r} C_{\substack{q u \\
\text { vwst }}}^{(8)}+\frac{1}{2 N_{c}}\left[Y_{u}\right]_{s v}\left[Y_{u}^{\dagger}\right]_{w t} C_{\substack{q u \\
v w r}}^{(8)}-\frac{1}{2}\left[Y_{u}^{\dagger}\right]_{w r}\left[Y_{u}\right]_{s v} C_{\substack{q u p t \\
v w p}}^{(8)}-\frac{1}{2}\left[Y_{u}^{\dagger}\right]_{w t}\left[Y_{u}\right]_{p v} C_{\substack{q u \\
v w r}}^{(8)}
\end{aligned}
$$

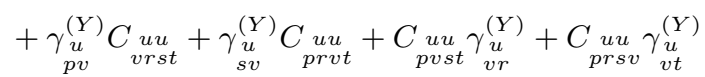

$$
\begin{aligned}
& \dot{C}_{p r s t}=\left[Y_{d} Y_{d}^{\dagger}\right]_{p r} C_{H_{s t}}+\left[Y_{d} Y_{d}^{\dagger}\right]_{s t} C_{p d}-\left[Y_{d}^{\dagger}\right]_{w r}\left[Y_{d}\right]_{p v} C_{\substack{q d \\
v w s t}}^{(1)}-\left[Y_{d}^{\dagger}\right]_{w t}\left[Y_{d}\right]_{s v} C_{\substack{q d \\
v w r}}^{(1)} \\
& +\frac{1}{2 N_{c}}\left[Y_{d}\right]_{p v}\left[Y_{d}^{\dagger}\right]_{w r} C_{\substack{q d \\
\text { vwst }}}^{(8)}+\frac{1}{2 N_{c}}\left[Y_{d}\right]_{s v}\left[Y_{d}^{\dagger}\right]_{w t} C_{\begin{array}{c}
q d \\
\text { vwpr }
\end{array}}^{(8)}-\frac{1}{2}\left[Y_{d}^{\dagger}\right]_{w r}\left[Y_{d}\right]_{s v} C_{\begin{array}{c}
q d \\
v w p t
\end{array}}^{(8)}-\frac{1}{2}\left[Y_{d}^{\dagger}\right]_{w t}\left[Y_{d}\right]_{p v} C_{\begin{array}{c}
q d \\
v w s
\end{array}}^{(8)}
\end{aligned}
$$

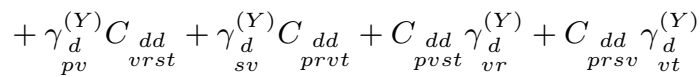

$$
\begin{aligned}
& \dot{C}_{p r s t}^{e u}=2\left[Y_{e} Y_{e}^{\dagger}\right]_{p r} C_{H_{s t}}-2\left[Y_{u} Y_{u}^{\dagger}\right]_{s t} C_{\text {pre }}+\left(\left[Y_{e}\right]_{p v}\left[Y_{u}\right]_{s w} C_{\substack{l e q u \\
\text { vrwt }}}^{(1)}+\left[Y_{e}^{\dagger}\right]_{v r}\left[Y_{u}^{\dagger}\right]_{w t} C_{\substack{l e q u \\
\text { vpws }}}^{(1) *}\right) \\
& -2\left[Y_{e}\right]_{p v}\left[Y_{e}^{\dagger}\right]_{w r} C_{v w s t}^{l u}-12\left(\left[Y_{e}\right]_{p v}\left[Y_{u}\right]_{s w} C_{\text {lequ }}^{(3)}+\left[Y_{e}^{\dagger}\right]_{v r}\left[Y_{u}^{\dagger}\right]_{w t} C_{\text {lequ }}^{(3) *}\right)
\end{aligned}
$$

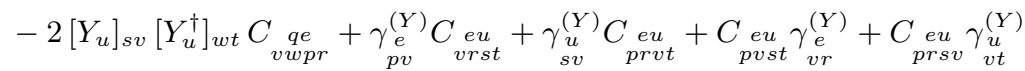

$$
\begin{aligned}
& \dot{C}_{p e d}=2\left[Y_{e} Y_{e}^{\dagger}\right]_{p r} C_{\text {prd }}+2\left[Y_{d} Y_{d}^{\dagger}\right]_{s t} C_{p r}-2\left[Y_{e}\right]_{p v}\left[Y_{e}^{\dagger}\right]_{w r} C_{v w s t}^{l d}-2\left[Y_{d}\right]_{s v}\left[Y_{d}^{\dagger}\right]_{w t} C_{v w p r}^{q e}
\end{aligned}
$$

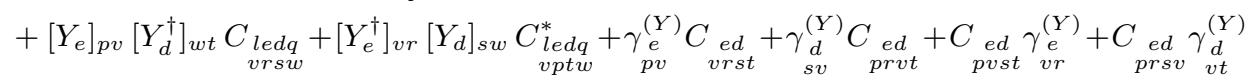

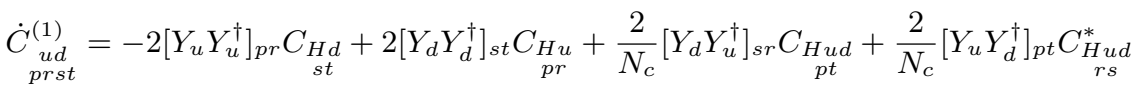

$$
\begin{aligned}
& +\frac{1}{N_{c}}\left(\left[Y_{d}\right]_{s v}\left[Y_{u}\right]_{p w} C_{\substack{q u d d \\
v r w t}}^{(1)}+\left[Y_{d}^{\dagger}\right]_{v t}\left[Y_{u}^{\dagger}\right]_{w r} C_{\substack{q u q d \\
v p w s}}^{(1) *}\right)-\left[Y_{d}\right]_{s w}\left[Y_{u}\right]_{p v} C_{\substack{q u q d \\
v r w t}}^{(1)}-\left[Y_{d}^{\dagger}\right]_{w t}\left[Y_{u}^{\dagger}\right]_{v r} C_{\substack{q u q d \\
\text { vpws }}}^{(1) *} \\
& +\frac{N_{c}^{2}-1}{2 N_{c}^{2}}\left(\left[Y_{d}\right]_{s v}\left[Y_{u}\right]_{p w} C_{q u q d}^{(8)}+\left[Y_{d}^{\dagger}\right]_{v t}\left[Y_{u}^{\dagger}\right]_{w r} C_{\substack{q u d \\
v p w s}}^{(8) *}\right)-2\left[Y_{u}\right]_{p v}\left[Y_{u}^{\dagger}\right]_{w r} C_{\substack{q d \\
v w s}}^{(1)}-2\left[Y_{d}\right]_{s v}\left[Y_{d}^{\dagger}\right]_{w t} C_{\substack{q u \\
v w p}}^{(1)}
\end{aligned}
$$

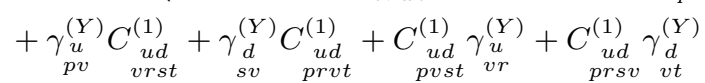

$$
\begin{aligned}
& \underset{p r s t}{\dot{C}_{p d}^{(8)}}=4\left[Y_{d} Y_{u}^{\dagger}\right]_{s r} C_{H_{p t}}+4\left[Y_{u} Y_{d}^{\dagger}\right]_{p t} C_{H u d}^{*}+2\left(\left[Y_{d}\right]_{s v}\left[Y_{u}\right]_{p w} C_{\substack{q u q d \\
\text { vrwt }}}^{(1)}+\left[Y_{d}^{\dagger}\right]_{v t}\left[Y_{u}^{\dagger}\right]_{w r} C_{\begin{array}{c}
q \text { qud } \\
\text { vpws }
\end{array}}^{(1) *}\right)
\end{aligned}
$$




$$
\begin{aligned}
& -2\left[Y_{u}\right]_{p v}\left[Y_{u}^{\dagger}\right]_{w r} C_{\substack{q d \\
\text { vwst }}}^{(8)}-2\left[Y_{d}\right]_{s v}\left[Y_{d}^{\dagger}\right]_{w t} C_{\substack{q u p r \\
v w p}}^{(8)}-\frac{1}{N_{c}}\left(\left[Y_{d}\right]_{s v}\left[Y_{u}\right]_{p w} C_{\substack{q u q d \\
v r w t}}^{(8)}+\left[Y_{d}^{\dagger}\right]_{v t}\left[Y_{u}^{\dagger}\right]_{w r} C_{\substack{q u q d \\
v p w s}}^{(8) *}\right)
\end{aligned}
$$

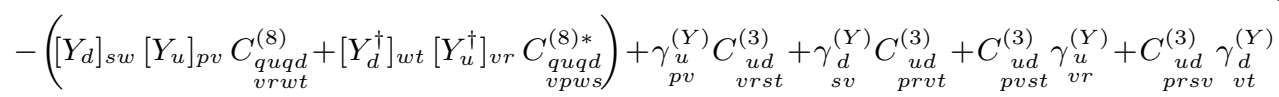

\section{A.8.3 $(\bar{L} L)(\bar{R} R)$}

$$
\begin{aligned}
& \dot{C}_{p r s t}^{l e}=\left[Y_{e}\right]_{s r} \xi_{p t}-\left[Y_{e}^{\dagger} Y_{e}\right]_{p r} C_{s t}+2\left[Y_{e} Y_{e}^{\dagger}\right]_{s t} C_{j l}^{(1)}-\left[Y_{e r}^{\dagger}\right]_{p v}\left[Y_{e}\right]_{w r} C_{v t s w}^{e e}-\left[Y_{e}^{\dagger}\right]_{p w}\left[Y_{e}\right]_{v r} C_{w t s v}^{e e} \\
& -2\left[Y_{e}^{\dagger}\right]_{p v}\left[Y_{e}\right]_{w r} C_{v w s t}^{e e}+\left[Y_{e}^{\dagger}\right]_{p w}\left[Y_{e}\right]_{s v} C_{v r w t}^{l e}-\left[Y_{e}^{\dagger}\right]_{w t}\left[Y_{e}\right]_{s v} C_{p w v r}^{l l}-\left[Y_{e}^{\dagger}\right]_{v t}\left[Y_{e}\right]_{s w} C_{p v w r}^{u l} \\
& -4\left[Y_{e}^{\dagger}\right]_{w t}\left[Y_{e}\right]_{s v} C_{p r v w}^{l l}+\left[Y_{e}^{\dagger}\right]_{v t}\left[Y_{e}\right]_{w r} C_{p v s w}^{l e}+\gamma_{p v}^{(Y)} C_{v r s t}^{l e}+\gamma_{s v}^{(Y)} C_{p r v t}^{l e}+C_{p v s t} \gamma_{v r}^{(Y)}+C_{p r s v} \gamma_{v t}^{(Y)} \gamma_{v e}^{(Y)}
\end{aligned}
$$

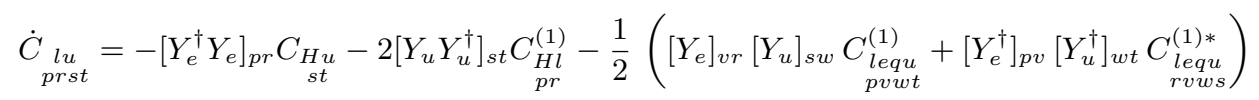

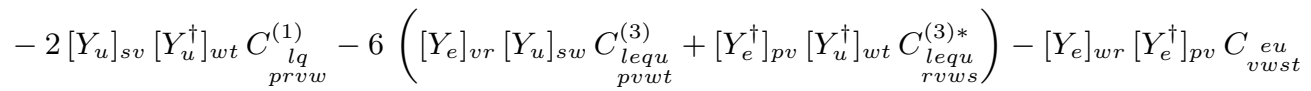

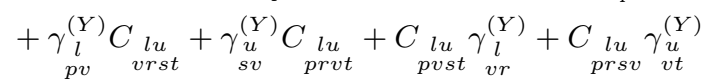

$$
\begin{aligned}
& \dot{C}_{p r s t}^{l d}=-\left[Y_{e}^{\dagger} Y_{e}\right]_{p r} C_{s t}+2\left[Y_{d} Y_{d}^{\dagger}\right]_{s t} C_{p r}^{(1)}-\frac{1}{2}\left(\left[Y_{e}\right]_{v r}\left[Y_{d}^{\dagger}\right]_{w t} C_{\substack{\text { ledq } \\
\text { pvsw }}}+\left[Y_{e}^{\dagger}\right]_{p v}\left[Y_{d}\right]_{s w} C_{\text {ledq }}^{*}\right)
\end{aligned}
$$

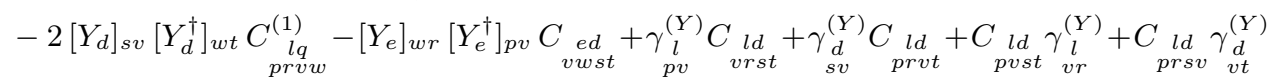

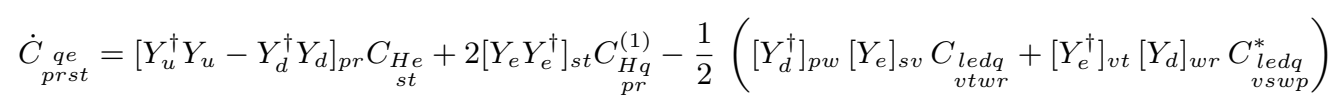

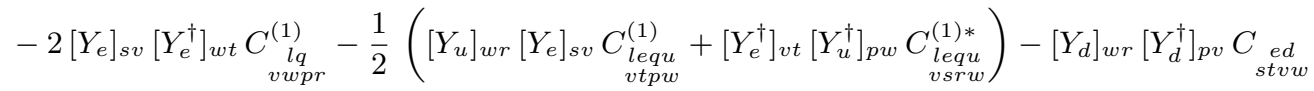

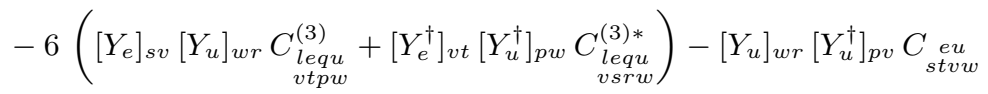

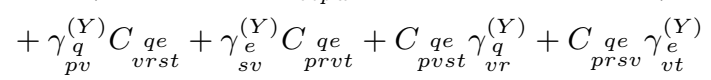

$$
\begin{aligned}
& \underset{\dot{C}_{q u}}{\dot{p}_{p t}^{(1)}}=\frac{1}{N_{c}}\left[Y_{u}\right]_{s r} \xi_{p t}+\left[Y_{u}^{\dagger} Y_{u}-Y_{d}^{\dagger} Y_{d}\right]_{p r} C_{H_{s t}}-2\left[Y_{u} Y_{u}^{\dagger}\right]_{s t} C_{\substack{H q \\
p r}}^{(1)} \\
& +\frac{1}{N_{c}}\left(\left[Y_{u}^{\dagger}\right]_{p w}\left[Y_{u}\right]_{s v} C_{\substack{q u \\
\text { vrwt }}}^{(1)}+\left[Y_{u}^{\dagger}\right]_{v t}\left[Y_{u}\right]_{w r} C_{\substack{q u \\
p v s w}}^{(1)}+\left[Y_{d}\right]_{w r}\left[Y_{u}\right]_{s v} C_{\substack{q u q d \\
p t v w}}^{(1)}+\left[Y_{d}^{\dagger}\right]_{p w}\left[Y_{u}^{\dagger}\right]_{v t} C_{\substack{q u d \\
r s v w}}^{(1) *}\right) \\
& -\frac{1}{2 N_{c}^{2}}\left(\left[Y_{u}^{\dagger}\right]_{p w}\left[Y_{u}\right]_{s v} C_{\substack{q u \\
v r w t}}^{(8)}+\left[Y_{u}^{\dagger}\right]_{v t}\left[Y_{u}\right]_{w r} C_{\substack{q u \\
p v s w}}^{(8)}+\left[Y_{d}\right]_{w r}\left[Y_{u}\right]_{s v} C_{\substack{q u q d \\
p t v w}}^{(8)}+\left[Y_{d}^{\dagger}\right]_{p w}\left[Y_{u}^{\dagger}\right]_{v t} C_{\substack{q u q d \\
r s v w}}^{(8) *}\right) \\
& -\frac{1}{N_{c}}\left(\left[Y_{u}^{\dagger}\right]_{v t}\left[Y_{u}\right]_{s w} C_{p v w r}^{(1)}+\left[Y_{u}^{\dagger}\right]_{w t}\left[Y_{u}\right]_{s v} C_{p w w r}^{(1)}+\left[Y_{u}^{\dagger}\right]_{p v}\left[Y_{u}\right]_{w r} C_{v t s w}^{u u}+\left[Y_{u}^{\dagger}\right]_{p w}\left[Y_{u}\right]_{v r} C_{w u t s v} \underset{w u}{w t s v}\right) \\
& -\frac{3}{N_{c}}\left(\left[Y_{u}^{\dagger}\right]_{v t}\left[Y_{u}\right]_{s w} C_{\substack{q q \\
p v w r}}^{(3)}+\left[Y_{u}^{\dagger}\right]_{w t}\left[Y_{u}\right]_{s v} C_{\substack{q q \\
p w v r}}^{(3)}\right)+\frac{1}{2}\left(\left[Y_{u}^{\dagger}\right]_{p w}\left[Y_{u}\right]_{s v} C_{\substack{q u \\
v r w t}}^{(8)}+\left[Y_{u}^{\dagger}\right]_{v t}\left[Y_{u}\right]_{w r} C_{\substack{q u \\
p v s w}}^{(8)}\right) \\
& +\frac{1}{2}\left(\left[Y_{u}\right]_{s v}\left[Y_{d}\right]_{w r} C_{\substack{q u d \\
v t p w}}^{(1)}+\left[Y_{d}^{\dagger}\right]_{p w}\left[Y_{u}^{\dagger}\right]_{v t} C_{\substack{q u q d \\
\text { vsrw }}}^{(1) *}+\left[Y_{u}\right]_{s v}\left[Y_{d}\right]_{w r} C_{\substack{q u d \\
\text { ptvw }}}^{(8)}+\left[Y_{d}^{\dagger}\right]_{p w}\left[Y_{u}^{\dagger}\right]_{v t} C_{\substack{q u q d \\
r s v w}}^{(8) *}\right) \\
& -4\left[Y_{u}^{\dagger}\right]_{w t}\left[Y_{u}\right]_{s v} C_{\substack{q q \\
p r v w}}^{(1)}-2\left[Y_{u}^{\dagger}\right]_{p v}\left[Y_{u}\right]_{w r} C_{v w u s t} u-\left[Y_{d}^{\dagger}\right]_{p v}\left[Y_{d}\right]_{w r} C_{\substack{u d \\
\text { stvw }}}^{(1)}
\end{aligned}
$$

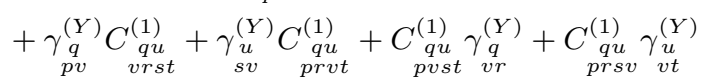

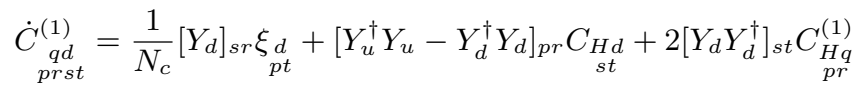




$$
\begin{aligned}
& +\frac{1}{N_{c}}\left(\left[Y_{d}^{\dagger}\right]_{p w}\left[Y_{d}\right]_{s v} C_{\substack{q d \\
\text { vrwt }}}^{(1)}+\left[Y_{d}^{\dagger}\right]_{v t}\left[Y_{d}\right]_{w r} C_{\substack{q d \\
\text { pvsw }}}^{(1)}+\left[Y_{u}\right]_{w r}\left[Y_{d}\right]_{s v} C_{\substack{q u q d \\
\text { vwpt }}}^{(1)}+\left[Y_{u}^{\dagger}\right]_{p w}\left[Y_{d}^{\dagger}\right]_{v t} C_{\begin{array}{c}
\text { qudd } \\
\text { vwrs }
\end{array}}^{(1) *}\right) \\
& -\frac{1}{2 N_{c}^{2}}\left(\left[Y_{d}^{\dagger}\right]_{p w}\left[Y_{d}\right]_{s v} C_{\substack{q d \\
v r w t}}^{(8)}+\left[Y_{d}^{\dagger}\right]_{v t}\left[Y_{d}\right]_{w r} C_{\substack{q d \\
p v s w}}^{(8)}+\left[Y_{u}\right]_{w r}\left[Y_{d}\right]_{s v} C_{q u q d}^{(8)}+\left[Y_{u w p t}^{\dagger}\right]_{p w}\left[Y_{d}^{\dagger}\right]_{v t} C_{\substack{q u d \\
v w r s}}^{(8) *}\right) \\
& -\frac{1}{N_{c}}\left(\left[Y_{d}^{\dagger}\right]_{v t}\left[Y_{d}\right]_{s w} C_{p v w r}^{(1)}+\left[Y_{d}^{\dagger}\right]_{w t}\left[Y_{d}\right]_{s v} \underset{p w w r}{C_{p q u}^{(1)}}+\left[Y_{d}^{\dagger}\right]_{p v}\left[Y_{d}\right]_{w r} C_{v t s w}^{d d}+\left[Y_{d}^{\dagger}\right]_{p w}\left[Y_{d}\right]_{v r} C_{w t s v}^{d d}\right)
\end{aligned}
$$

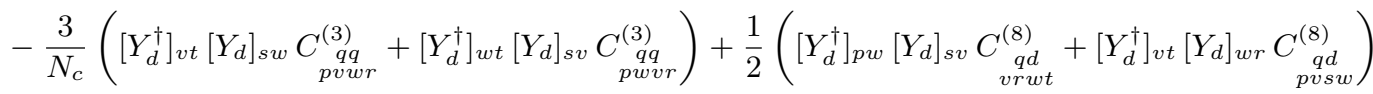

$$
\begin{aligned}
& +\frac{1}{2}\left(\left[Y_{d}\right]_{s w}\left[Y_{u}\right]_{v r} C_{\substack{q u q d \\
\text { pvwt }}}^{(1)}+\left[Y_{u}^{\dagger}\right]_{p v}\left[Y_{d}^{\dagger}\right]_{w t} C_{\substack{q u q d \\
r v w s}}^{(1) *}+\left[Y_{d}\right]_{s v}\left[Y_{u}\right]_{w r} C_{\substack{q u d \\
\text { vwpt }}}^{(8)}+\left[Y_{u}^{\dagger}\right]_{p w}\left[Y_{d}^{\dagger}\right]_{v t} C_{\substack{q u q d \\
\text { vwrs }}}^{(8) *}\right) \\
& -4\left[Y_{d}^{\dagger}\right]_{w t}\left[Y_{d}\right]_{s v} C_{\substack{q q \\
p r v w}}^{(1)}-2\left[Y_{d}^{\dagger}\right]_{p v}\left[Y_{d}\right]_{w r} C_{v w d} d x-\left[Y_{u}^{\dagger}\right]_{p v}\left[Y_{u}\right]_{w r} C_{\substack{u d \\
v w s t}}^{(1)}
\end{aligned}
$$

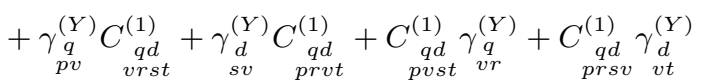

$$
\begin{aligned}
& \dot{C}_{p r s t}^{(8)}=2\left[Y_{u}\right]_{s r} \xi_{p t}^{u} \\
& -\frac{1}{N_{c}}\left(\left[Y_{u}^{\dagger}\right]_{p w}\left[Y_{u}\right]_{s v} C_{\substack{q u \\
\text { vrwt }}}^{(8)}+\left[Y_{u}^{\dagger}\right]_{v t}\left[Y_{u}\right]_{w r} C_{\substack{q u \\
p v s w}}^{(8)}+\left[Y_{d}\right]_{w r}\left[Y_{u}\right]_{s v} C_{\substack{q u q d \\
p t v w}}^{(8)}+\left[Y_{d}^{\dagger}\right]_{p w}\left[Y_{u}^{\dagger}\right]_{v t} C_{\substack{q u d d \\
r s v w}}^{(8) *}\right) \\
& +2\left(\left[Y_{u}\right]_{s v}\left[Y_{d}\right]_{w r} C_{\substack{q u q d \\
p t v w}}^{(1)}+\left[Y_{u}^{\dagger}\right]_{v t}\left[Y_{d}^{\dagger}\right]_{p w} C_{\substack{q u q d \\
r s v w}}^{(1) *}+\frac{1}{4}\left[Y_{u}\right]_{s v}\left[Y_{d}\right]_{w r} C_{\substack{q u q d \\
v t p w}}^{(8)}+\frac{1}{4}\left[Y_{u}^{\dagger}\right]_{v t}\left[Y_{d}^{\dagger}\right]_{p w} C_{\substack{q u q d \\
v s r w}}^{(8) *}\right) \\
& -2\left(\left[Y_{u}^{\dagger}\right]_{v t}\left[Y_{u}\right]_{s w} C_{\substack{q q \\
p v w r}}^{(1)}+\left[Y_{u}^{\dagger}\right]_{w t}\left[Y_{u}\right]_{s v} \underset{\substack{q q \\
p w r v}}{C_{p w}^{(1)}}-\left[Y_{u}^{\dagger}\right]_{p w}\left[Y_{u}\right]_{s v} C_{\substack{q u \\
\text { vrw }}}^{(1)}-\left[Y_{u}^{\dagger}\right]_{v t}\left[Y_{u}\right]_{w r} C_{\substack{q u \\
p v w}}^{(1)}\right) \\
& -2\left(\left[Y_{u}^{\dagger}\right]_{p v}\left[Y_{u}\right]_{w r} C_{v t s w}^{u u}+\left[Y_{u}^{\dagger}\right]_{p w}\left[Y_{u}\right]_{v r} C_{w t s v}^{u u}\right)-6\left(\left[Y_{u}^{\dagger}\right]_{v t}\left[Y_{u}\right]_{s w} C_{p v w r}^{(3)}+\left[Y_{u}^{\dagger}\right]_{w t}\left[Y_{u}\right]_{s v} C_{p w q u}^{(3)}\right)
\end{aligned}
$$

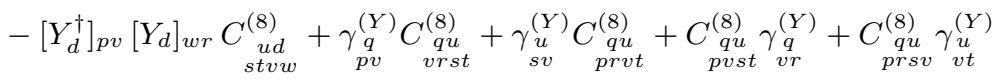

$$
\begin{aligned}
& \underset{p r s t}{\dot{C}_{p d}^{(8)}}=2\left[Y_{d}\right]_{s r} \xi_{d}
\end{aligned}
$$

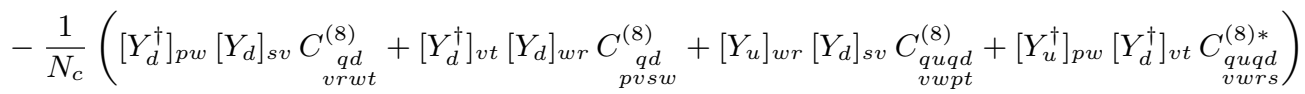

$$
\begin{aligned}
& +2\left(\left[Y_{d}\right]_{s v}\left[Y_{u}\right]_{w r} C_{\substack{q u q d \\
\text { vwpt }}}^{(1)}+\left[Y_{d}^{\dagger}\right]_{v t}\left[Y_{u}^{\dagger}\right]_{p w} C_{\substack{q u q d \\
\text { vwrs }}}^{(1) *}+\frac{1}{4}\left[Y_{u}\right]_{v r}\left[Y_{d}\right]_{s w} C_{\substack{q u q d \\
\text { pvwt }}}^{(8)}+\frac{1}{4}\left[Y_{u}^{\dagger}\right]_{p v}\left[Y_{d}^{\dagger}\right]_{w t} C_{\begin{array}{c}
\text { quqd } \\
\text { rvws }
\end{array}}^{(8) *}\right)
\end{aligned}
$$

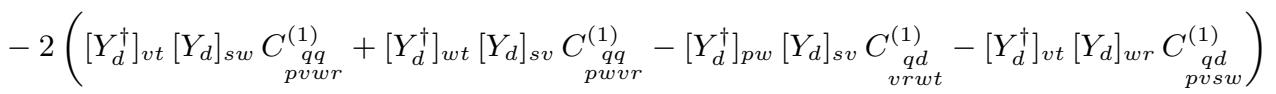

$$
\begin{aligned}
& -2\left(\left[Y_{d}^{\dagger}\right]_{p v}\left[Y_{d}\right]_{w r} C_{d d d}+\left[Y_{d}^{\dagger}\right]_{p w}\left[Y_{d}\right]_{v r} C_{w d d}^{d d}\right)-6\left(\left[Y_{d t s}^{\dagger}\right]_{v t}\left[Y_{d}\right]_{s w} C_{\substack{q q \\
p v w}}^{(3)}+\left[Y_{d}^{\dagger}\right]_{w t}\left[Y_{d}\right]_{s v} C_{\substack{q q \\
p w v r}}^{(3)}\right)
\end{aligned}
$$

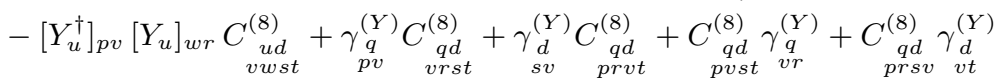

\section{A.8.4 $(\bar{L} R)(\bar{R} L)$}

$$
\begin{aligned}
& \dot{C}_{\text {ledq }}=-2\left[Y_{d}\right]_{s t} \xi_{p r}-2\left[Y_{e}\right]_{p r}^{*} \xi_{s t}^{*}+2\left[Y_{e}^{\dagger}\right]_{p v}\left[Y_{d}\right]_{w t} C_{v r s w}^{\text {ed }}-2\left[Y_{e}^{\dagger}\right]_{v r}\left[Y_{d}\right]_{w t} C_{p v s w}^{l d}+2\left[Y_{e}^{\dagger}\right]_{v r}\left[Y_{d}\right]_{s w} C_{p v w t}^{(1)} \\
& +6\left[Y_{e}^{\dagger}\right]_{v r}\left[Y_{d}\right]_{s w} C_{\substack{l q \\
\text { pvt }}}^{(3)}-2\left[Y_{e}^{\dagger}\right]_{p w}\left[Y_{d}\right]_{s v} C_{v t w r}^{q e}+2\left[Y_{d}\right]_{s v}\left[Y_{u}\right]_{w t} C_{\text {lequ }}^{(1)}
\end{aligned}
$$

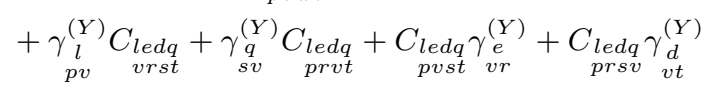

\section{A.8.5 $(\bar{L} R)(\bar{L} R)$}

$$
\underset{\dot{C}_{l e q u}}{\dot{p r s t}^{(1)}}=2\left[Y_{u}^{\dagger}\right]_{s t} \xi_{p r}+2\left[Y_{e}^{\dagger}\right]_{p r} \xi_{s t}^{u}+2\left[Y_{d}^{\dagger}\right]_{s v}\left[Y_{u}^{\dagger}\right]_{w t} C_{\text {ledq }}+2\left[Y_{e}^{\dagger}\right]_{p v}\left[Y_{u}^{\dagger}\right]_{s w} C_{v r w t}+2\left[Y_{e}^{\dagger}\right]_{v r}\left[Y_{u}^{\dagger}\right]_{w t} C_{p v s w}^{(1)}
$$




$$
\begin{aligned}
& -6\left[Y_{e}^{\dagger}\right]_{v r}\left[Y_{u}^{\dagger}\right]_{w t} C_{p v s w}^{(3)}-2\left[Y_{e}^{\dagger}\right]_{v r}\left[Y_{u}^{\dagger}\right]_{s w} C_{p v w t}{ }_{p v w}-2\left[Y_{e}^{\dagger}\right]_{p w}\left[Y_{u}^{\dagger}\right]_{v t} C_{s v w r}^{q e}
\end{aligned}
$$

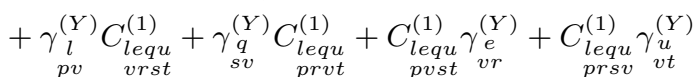

$$
\begin{aligned}
& \underset{\substack{l e q u \\
\text { prst }}}{\dot{x}^{(3)}}=-\frac{1}{2}\left[Y_{u}^{\dagger}\right]_{s w}\left[Y_{e}^{\dagger}\right]_{p v} C_{\text {vrwt }}^{e u}-\frac{1}{2}\left[Y_{e}^{\dagger}\right]_{v r}\left[Y_{u}^{\dagger}\right]_{w t} C_{\substack{l q \\
\text { pvsw }}}^{(1)}+\frac{3}{2}\left[Y_{e}^{\dagger}\right]_{v r}\left[Y_{u}^{\dagger}\right]_{w t} C_{\substack{l q \\
\text { pvsw }}}^{(3)} \\
& -\frac{1}{2}\left[Y_{e}^{\dagger}\right]_{v r}\left[Y_{u}^{\dagger}\right]_{s w} C_{p v w t}^{l u}-\frac{1}{2}\left[Y_{e}^{\dagger}\right]_{p w}\left[Y_{u}^{\dagger}\right]_{v t} C_{s v w r}^{q e}
\end{aligned}
$$

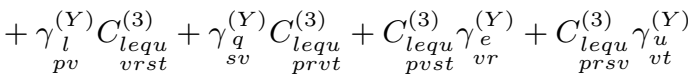

$$
\begin{aligned}
& \dot{C}_{\substack{q u q d \\
p r s t}}^{(1)}=-2\left[Y_{u}^{\dagger}\right]_{p r} \xi_{\text {st }}-2\left[Y_{d}^{\dagger}\right]_{s t} \xi_{p r} \\
& -\frac{2}{N_{c}^{2}}\left(\left[Y_{u}^{\dagger}\right]_{v r}\left[Y_{d}^{\dagger}\right]_{p w} C_{\substack{q d \\
\text { svwt }}}^{(8)}+\left[Y_{d}^{\dagger}\right]_{v t}\left[Y_{u}^{\dagger}\right]_{s w} C_{\substack{q u \\
p v w r}}^{(8)}+\left[Y_{d}^{\dagger}\right]_{p w}\left[Y_{u}^{\dagger}\right]_{s v} \underset{\substack{u d \\
v r w t}}{C^{(8)}}\right) \\
& +\frac{4}{N_{c}}\left(\left[Y_{d}^{\dagger}\right]_{w t}\left[Y_{u}^{\dagger}\right]_{v r} C_{\substack{q q \\
\text { svpw }}}^{(1)}+\left[Y_{d}^{\dagger}\right]_{v t}\left[Y_{u}^{\dagger}\right]_{w r} C_{\substack{q q \\
\text { pvsw }}}^{(1)}-3\left[Y_{d}^{\dagger}\right]_{w t}\left[Y_{u}^{\dagger}\right]_{v r} C_{\substack{q u \\
\text { svp }}}^{(3)}-3\left[Y_{d}^{\dagger}\right]_{v t}\left[Y_{u}^{\dagger}\right]_{w r} C_{\substack{q q \\
p v s w}}^{(3)}\right) \\
& +\frac{4}{N_{c}}\left(\left[Y_{d}^{\dagger}\right]_{p w}\left[Y_{u}^{\dagger}\right]_{v r} C_{\substack{q d \\
s v w t}}^{(1)}+\left[Y_{d}^{\dagger}\right]_{v t}\left[Y_{u}^{\dagger}\right]_{s w} C_{\substack{q u \\
p v w r}}^{(1)}+\left[Y_{d}^{\dagger}\right]_{p w}\left[Y_{u}^{\dagger}\right]_{s v} \underset{\substack{u d \\
v r w t}}{C^{(1)}}\right) \\
& -4\left(\left[Y_{d}^{\dagger}\right]_{w t}\left[Y_{u}^{\dagger}\right]_{v r} C_{\substack{q q \\
p v s w}}^{(1)}+\left[Y_{d}^{\dagger}\right]_{v t}\left[Y_{u}^{\dagger}\right]_{w r} C_{\substack{q q \\
\text { svpw }}}^{(1)}\right)+12\left(\left[Y_{d}^{\dagger}\right]_{w t}\left[Y_{u}^{\dagger}\right]_{v r} \underset{p v s q}{C_{p q u}^{(3)}}+\left[Y_{d}^{\dagger}\right]_{v t}\left[Y_{u}^{\dagger}\right]_{w r} C_{\substack{q q \\
\text { svpw }}}^{(3)}\right) \\
& +2\left(\left[Y_{d}^{\dagger}\right]_{p w}\left[Y_{u}^{\dagger}\right]_{v r} C_{\substack{q d \\
\text { svwt }}}^{(8)}+\left[Y_{d}^{\dagger}\right]_{v t}\left[Y_{u}^{\dagger}\right]_{s w} C_{\substack{q u w r \\
p v w}}^{(8)}+\left[Y_{d}^{\dagger}\right]_{p w}\left[Y_{u}^{\dagger}\right]_{s v} C_{\substack{u d \\
v r w}}^{(8)}\right)-4\left[Y_{d}^{\dagger}\right]_{s w}\left[Y_{u}^{\dagger}\right]_{p v} C_{\substack{u d \\
v r w t}}^{(1)}
\end{aligned}
$$

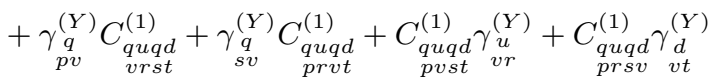

$$
\begin{aligned}
& \dot{C}_{\substack{q u q d \\
p r s t}}^{(8)}=-\frac{4}{N_{c}}\left(\left[Y_{d}^{\dagger}\right]_{p w}\left[Y_{u}^{\dagger}\right]_{v r} C_{\substack{q d \\
\text { svwt }}}^{(8)}+\left[Y_{d}^{\dagger}\right]_{v t}\left[Y_{u}^{\dagger}\right]_{s w} C_{\substack{q u w r \\
p v w}}^{(8)}+\left[Y_{d}^{\dagger}\right]_{p w}\left[Y_{u}^{\dagger}\right]_{s v} C_{\substack{u d \\
v r w t}}^{(8)}\right) \\
& +8\left(\left[Y_{d}^{\dagger}\right]_{w t}\left[Y_{u}^{\dagger}\right]_{v r} C_{\substack{q q \\
\text { svpw }}}^{(1)}+\left[Y_{d}^{\dagger}\right]_{v t}\left[Y_{u}^{\dagger}\right]_{w r} C_{\substack{q q \\
p v s w}}^{(1)}\right)-24\left(\left[Y_{d}^{\dagger}\right]_{w t}\left[Y_{u}^{\dagger}\right]_{v r} C_{\substack{q q \\
\text { svpw }}}^{(3)}+\left[Y_{d}^{\dagger}\right]_{v t}\left[Y_{u}^{\dagger}\right]_{w r} C_{\substack{q q \\
p v s w}}^{(3)}\right) \\
& +8\left(\left[Y_{d}^{\dagger}\right]_{p w}\left[Y_{u}^{\dagger}\right]_{v r} C_{\substack{q d \\
\text { swwt }}}^{(1)}+\left[Y_{d}^{\dagger}\right]_{v t}\left[Y_{u}^{\dagger}\right]_{s w} C_{\substack{q u w r \\
p v w}}^{(1)}+\left[Y_{d}^{\dagger}\right]_{p w}\left[Y_{u}^{\dagger}\right]_{s v} C_{\substack{u d \\
v r w t}}^{(1)}\right)-4\left[Y_{d}^{\dagger}\right]_{s w}\left[Y_{u}^{\dagger}\right]_{p v} C_{\substack{u d \\
v r w t}}^{(8)}
\end{aligned}
$$

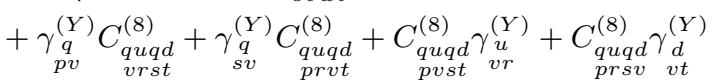

Open Access. This article is distributed under the terms of the Creative Commons Attribution License (CC-BY 4.0), which permits any use, distribution and reproduction in any medium, provided the original author(s) and source are credited.

\section{References}

[1] W. Buchmüller and D. Wyler, Effective Lagrangian analysis of new interactions and flavor conservation, Nucl. Phys. B 268 (1986) 621 [INSPIRE].

[2] B. Grzadkowski, M. Iskrzynski, M. Misiak and J. Rosiek, Dimension-six terms in the Standard Model Lagrangian, JHEP 10 (2010) 085 [arXiv: 1008.4884] [INSPIRE].

[3] C. Grojean, E.E. Jenkins, A.V. Manohar and M. Trott, Renormalization group scaling of Higgs operators and $\Gamma(h \rightarrow \gamma \gamma)$, JHEP 04 (2013) 016 [arXiv: 1301.2588] [INSPIRE].

[4] A.V. Manohar, An exactly solvable model for dimension six Higgs operators and $h \rightarrow \gamma \gamma$, Phys. Lett. B 726 (2013) 347 [arXiv:1305.3927] [INSPIRE]. 
[5] E.E. Jenkins, A.V. Manohar and M. Trott, Renormalization group evolution of the Standard Model dimension six operators I: formalism and $\lambda$ dependence, JHEP 10 (2013) 087 [arXiv: 1308.2627] [INSPIRE].

[6] A. Brignole and A. Rossi, Anatomy and phenomenology of $\mu-\tau$ lepton flavor violation in the MSSM, Nucl. Phys. B 701 (2004) 3 [hep-ph/0404211] [InSPIRE].

[7] S. Khan, Renormalization group evolution of the non-unitary operator, Nucl. Phys. B 864 (2012) 38 [arXiv: 1203.5043] [INSPIRE].

[8] W. Dekens and J. de Vries, Renormalization group running of dimension-six sources of parity and time-reversal violation, JHEP 05 (2013) 149 [arXiv:1303.3156] [INSPIRE].

[9] M. Raidal et al., Flavour physics of leptons and dipole moments, Eur. Phys. J. C 57 (2008) 13 [arXiv:0801.1826] [INSPIRE].

[10] J. Elias-Miró, J. Espinosa, E. Masso and A. Pomarol, Renormalization of dimension-six operators relevant for the Higgs decays $h \rightarrow \gamma \gamma, \gamma Z$, JHEP 08 (2013) 033 [arXiv:1302.5661] [INSPIRE].

[11] J. Elias-Miró, J. Espinosa, E. Masso and A. Pomarol, Higgs windows to new physics through $D=6$ operators: constraints and one-loop anomalous dimensions, JHEP 11 (2013) 066 [arXiv: 1308.1879] [INSPIRE].

[12] A. Manohar and H. Georgi, Chiral quarks and the nonrelativistic quark model, Nucl. Phys. B 234 (1984) 189 [INSPIRE].

[13] E.E. Jenkins, A.V. Manohar and M. Trott, Naive dimensional analysis counting of gauge theory amplitudes and anomalous dimensions, Phys. Lett. B 726 (2013) 697 [arXiv: 1309.0819] [INSPIRE].

[14] G. Blankenburg, J. Ellis and G. Isidori, Flavour-changing decays of a $125 \mathrm{GeV}$ Higgs-like particle, Phys. Lett. B $\mathbf{7 1 2}$ (2012) 386 [arXiv:1202.5704] [INSPIRE].

[15] R. Harnik, J. Kopp and J. Zupan, Flavor violating Higgs decays, JHEP 03 (2013) 026 [arXiv: 1209.1397] [INSPIRE].

[16] E.E. Jenkins, A.V. Manohar and M. Trott, On gauge invariance and minimal coupling, JHEP 09 (2013) 063 [arXiv: 1305.0017] [INSPIRE].

[17] MEG collaboration, J. Adam et al., New constraint on the existence of the $\mu^{+} \rightarrow e^{+} \gamma$ decay, Phys. Rev. Lett. 110 (2013) 201801 [arXiv:1303.0754] [INSPIRE].

[18] R.S. Chivukula and H. Georgi, Composite technicolor Standard Model, Phys. Lett. B 188 (1987) 99 [INSPIRE].

[19] G. D'Ambrosio, G. Giudice, G. Isidori and A. Strumia, Minimal flavor violation: an effective field theory approach, Nucl. Phys. B 645 (2002) 155 [hep-ph/0207036] [INSPIRE].

[20] A.J. Buras, Minimal flavor violation, Acta Phys. Polon. B 34 (2003) 5615 [hep-ph/0310208] [INSPIRE]. 